\title{
Convergent Force Fields Organized in the Frog's Spinal Cord
}

\author{
Simon F. Giszter, Ferdinando A. Mussa-Ivaldi, and Emilio Bizzi \\ Department of Brain and Cognitive Sciences, Massachusetts Institute of Technology, Cambridge, Massachusetts \\ 02139-4307
}

\begin{abstract}
Microstimulation of the gray matter of the frog's spinal cord was used to elicit motor responses. Force responses were recorded with the frog's ankle clamped while EMG activity was monitored. The collections of force patterns elicited at different leg configurations were summarized as force fields. These force fields showed convergence to an equilibrium point. The equilibrium paths were calculated from the force fields with the leg clamped. These paths predicted free limb motion in $75 \%$ of trials.
\end{abstract}

The force fields were separated into active and prestimulation resting responses. The active force field responses had a fixed position equilibrium. These active force fields were modulated in amplitude over time, although the balance and orientations of forces in the pattern remained fixed. The active fields grouped into a few classes. These included both convergent and parallel fields.

The convergent force fields (CFFS) could be observed in deafferented preparations. Motoneuron (MN) activity underlying the force fields was marked using sulforhodamine. The marked activity covered several segments. Several simulations and MN stimulations show that topography, limb geometry, and random activation could not account for the results. It is likely that propriospinal interneurons distribute the activity that underlies the responses observed here.

Experiments showed that CFFs that resemble those elicited by microstimulation also underlie natural behaviors. The full variety of fields revealed by microstimulation was larger than the repertoire elicited by cutaneous stimulation. It was concluded that fixed-pattern force fields elicited in the spinal cord may be viewed as movement primitives. These force fields could form building blocks for more complex behaviors.

[Key words: spinal behavior, virtual trajectory, movement, spinal gray, spinal cord, muscle synergy, force field, equilibrium point]

A fundamental issue in motor control is the nature of the basic units of motor behavior and their control (see Windhorst, 1991). In this study, we address several questions related to this issue.

\footnotetext{
Received Apr. 10, 1992; revised July 22, 1992; accepted July 28, 1992.

We thank Eric Loeb and Paul Borghesani for sharing occasional frogs, and Margo Cantor, Sylvester Sczepankowsi, Pat Harlan, and David Smith for essential technical assistance. This research was supported by Office of Naval Research Grant N00014/88/K/0372 and NIH Grants NS09343 and AR26710.

Correspondence should be addressed to Dr. Emilio Bizzi, Department of Brain and Cognitive Sciences, E25-526, Massachusetts Institute of Technology, 77 Massachusetts Avenue, Cambridge, MA 02139-4307.

Copyright (C) 1993 Society for Neuroscience $0270-6474 / 93 / 130467-25 \$ 05.00 / 0$
}

(1) What functional motoneuron (MN) groupings or synergies can be revealed in the spinal cord through the use of microstimulation? (2) What do these synergies and their mechanical effects reveal about multijoint control strategies in the spinal cord? More specifically, how do these synergies relate to limb posture and movement parameters? (3) How are these synergies related to the fixed-action patterns that are organized in the spinal cord (such as flexion and wiping behaviors) and their underlying motor strategies?

To address some of these issues, we examined the motor output elicited from frogs whose spinal cord was surgically separated from the rest of the CNS. A large body of work supports the notion that a variety of complex mechanisms for organizing motor output reside in the spinal cord. Both locomotor pattern generation and phasic limb-positioning mechanisms have large portions of their circuitry in the spinal cord (Shik and Orlovsky, 1976; Grillner and Zangger, 1979; Stein, 1983; Bekoff, 1985; Grillner and Wallen, 1985; Alstermark and Sasaki, 1986; Koshland and Smith, 1989). The spinalized frog preparation is known to display a variety of movements and complex kinematic adjustments (Fukson et al., 1980; Gelfand et al., 1988; Berkinblit et al., 1989; Giszter et al., 1989; Schotland et al., 1989; Ostry et al., 1991). Thus, this preparation is suitable for the study of spinal mechanisms of motor control.

To examine the spinal cord's control of the limb of the frog, we have used microstimulation of the spinal gray. This technique has frequently been utilized in previous research for the analysis of spinal cord circuitry (e.g., Gustafsson and Jankowska, 1976). In this research, we utilized an apparatus and technique that allowed us to examine the patterns of isometric forces that act at the ankle as a result of microstimulation. These patterns resulted from the interaction of the mechanics of the limb and the contractions of sets of muscles (or synergies) activated by the microstimulation of the spinal cord. Such patterns of forces are a useful summary of the behavior of the limb. By expressing our results as a force field, we were able to examine some aspects of how the frog's spinal cord organizes multijoint posture and movement.

\section{Materials and Methods}

\subsection{Surgical procedures}

We performed microstimulation experiments on approximately 100 spinalized bullfrogs. All surgeries were performed under standard tricaine anesthesia. The spinal cord was transected at the level of the calamus scriptorius. The tectal and other anterior areas were destroyed. The lumbar spinal cord was exposed by removing the spinal arches of the fourth, fifth, and sixth vertebrae. In the same surgical session, we implanted electrodes with bipolar leads in 11 leg muscles. These elec- 

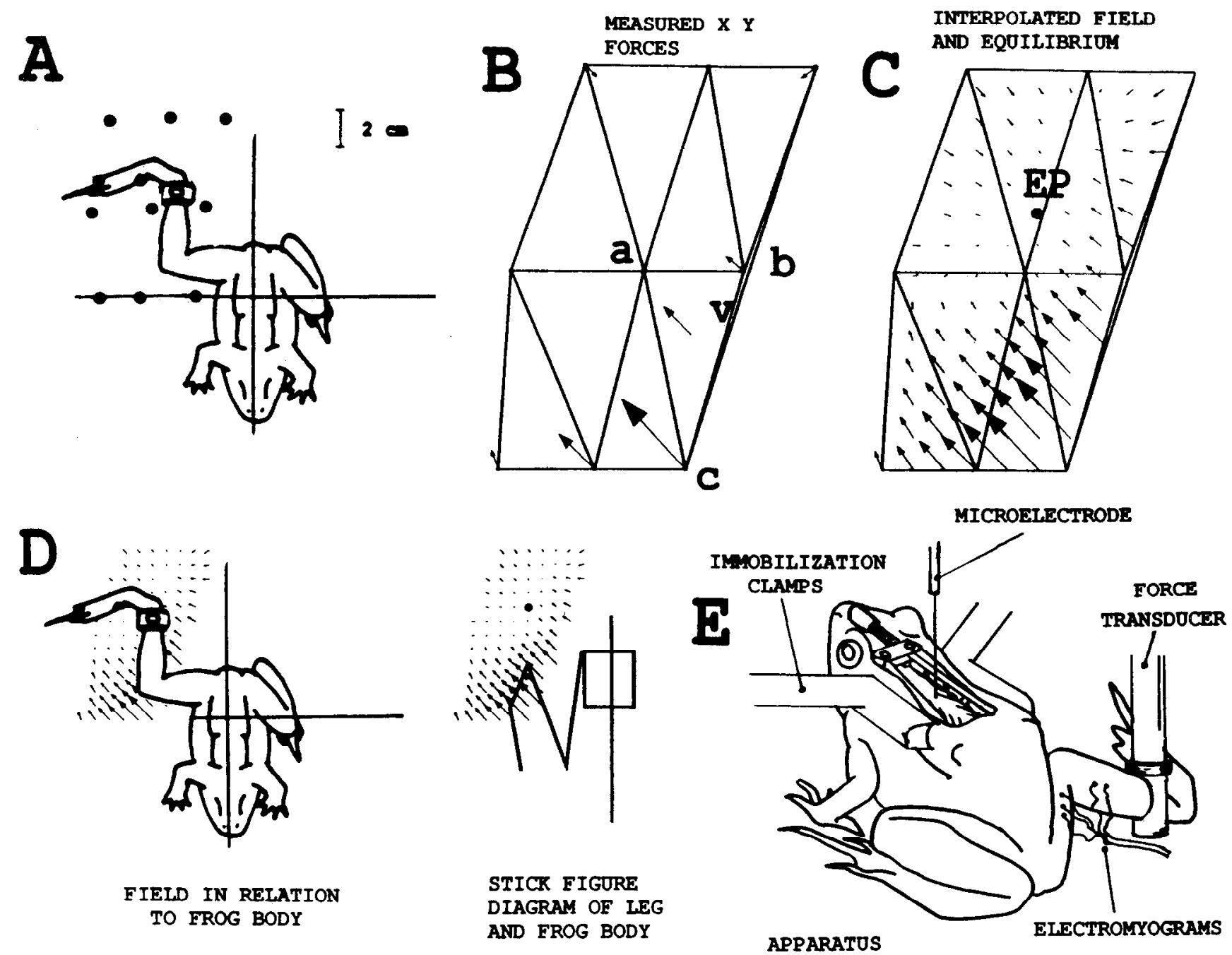

Figure 1. The apparatus and method of construction of a force field. $A$, A collection of forces is recorded at several different spatial locations (circles). B, A minimum perimeter (Delaunay) tessellation of the nine points is constructed. Within each triangle, vectors are estimated using an exact linear interpolation based on the three corner vectors. Thus, vector $\mathbf{v}$ is calculated using vectors at vertices a, $\mathbf{b}$, and $\mathbf{c}$. $C$, The interpolated field is used to find any equilibria $(E P)$. $D:$ Left, The interpolated force field is shown in relation to the frog in the apparatus. Right, This is reduced to a stylized construction that is used to express the relation of frog body axis and leg to the interpolated force field in the remaining figures. $E$, The apparatus. The spine is clamped. The pelvis is held clamped by restraints (not shown). With an electrode in the spinal gray, the mechanical response to stimulation is recorded at the force sensor that is attached to the limb at the ankle. The limb configuration is fully constrained by the pelvis restraint and the force sensor.

trodes allowed us to record electromyographic (EMG) activity from the following muscles: semitendinosus, vastus externus, vastus internus, rectus anticus, rectus internus, biceps, semimembranosus, gastrocnemius, tibialis anticus, sartorius, adductor magnus (Ecker, 1971). In five animals, dorsal roots 8,9 , and 10 were sectioned extradurally 3 weeks prior to spinal cord transection.

\subsection{Stimulation technique}

We elicited motor responses by microstimulating the spinal cord. Our microelectrodes were placed in what we estimated to be the lateral and intermediate neuropil zone, $500-1000 \mu \mathrm{m}$ in depth and 200-500 $\mu \mathrm{m}$ from the midline. We used stainless steel stimulating electrodes with impedances ranging from 1 to $10 \mathrm{M} \Omega$. We marked the locations of the electrodes with electrolytic lesions $(10 \mu \mathrm{A}, 15 \mathrm{sec})$, and then visualized these lesions and the associated iron deposition post-mortem by examining $30 \mu \mathrm{m}$ frozen sections stained with cresyl violet and Prussian blue. The lesions were found to cluster in the lateral neuropil area of the gray matter.

Each stimulus consisted of a train of anodal current impulses. Typically, the train lasted $300 \mathrm{msec}$. The monophasic anodal impulses had a duration of $1 \mathrm{msec}$ and a frequency of $40 \mathrm{~Hz}$. The current was controlled and delivered by a constant-current, linear-response stimulus isolator. The peak current's amplitude ranged between 1 and $8 \mu \mathrm{A}$. This peak magnitude corresponded to an estimated stimulation volume of less than $100 \mu \mathrm{m}$ radius (Ranck, 1981; Yeomans, 1990). Such values have been previously used in spinal cord physiology (e.g., Gustafsson and Jankowska, 1976). We chose this peak amplitude so as to elicit measurable mechanical responses while remaining close to the threshold of EMG activations.

\subsection{Data recording}

For each spinal stimulation, we collected the EMG signals from the implanted leg muscles and the mechanical force at the ankle. An IBMcompatible PC-386 was used to trigger the stimulation and to collect the data. Sampling frequencies were different for EMG and force. The EMG signals were sampled at a rate of $1 \mathrm{kHz}$ per channel. They were subsequently rectified and filtered off line with a time constant of 20 msec. The force data were collected at $100 \mathrm{~Hz}$.

To measure the mechanical responses, we attached the right ankle of the frog to a six-axis force transducer (ATI 310), as shown in Figure 1. The transducer held the ankle approximately level with the acetabulum. The transducer was mounted on a two-axis Cartesian manipulator. We set the Cartesian location of the frog's ankle (with a resolution of $1 \mathrm{~mm}$ ) using this manipulator. The $x-y$-plane corresponded approximately to 
the horizontal plane. The z-axis was directed upward orthogonally to the $x-y$-plane. The output of the transducer was a set of three forces and three torques; the components in each set lay along the $\mathrm{x}-, \mathrm{y}-$, and z-axes. The sensor's resolution was $0.01 \mathrm{~N}$ for the force components and $0.28 \mathrm{~N} \cdot \mathrm{mm}$ for the torque components. The sampling rate for the force signal was $100 \mathrm{~Hz}$. Although we sampled and stored all six force/ torque components, our subsequent analysis was limited to the $\mathrm{x}$ and $y$ translational components of the force vector.

\subsection{Marking synaptic activity using sulforhodamine}

In seven frogs, we examined the spread of activation through the cord following microstimulation. To track the spread, we used sulforhodamine. We bathed bilaterally deafferented animals in frog Ringer containing $0.5 \%$ sulforhodamine. The animals were then stimulated with the trains normally used in our experiments at a repeat rate of $0.05 \mathrm{~Hz}$ for $2 \mathrm{hr}$. Before and after this procedure, a single field was measured (see below). This procedure ensured that no significant clectrode movement had occurred during the sulforhodamine exposure and characterized the force field at the electrode site. The cord was then washed in vivo with a frog Ringer drip for $4 \mathrm{hr}$. The animal was perfused with buffered formaldehyde. Thirty-micron frozen sections were mounted and were examined for fluorescence in a Nikon fluorescence microscope and a Zeiss confocal video microscope.

The procedure was applied to three control animals with the following variations. The first control animal was not exposed to the $2 \mathrm{hr}, 0.05$ $\mathrm{Hz}$ stimulation during rhodamine application. The second control animal was stimulated at $0.05 \mathrm{~Hz}$ for only $1 \mathrm{hr}$. The third control animal was stimulated for the regular period while bathed in $10 \mathrm{~mm}$ Heptanol Ringer, which blocked gap junctions (Deleze and Herve, 1983; Bennett and Spray, 1987) and therefore the spread of sulforhodamine by this means. The controls confirmed that the uptake was stimulation dependent and varied in intensity with the stimulation's duration, but did not depend critically on gap junctions. Although the controls showed limited uptake at the cut end of the dorsal roots, the sulforhodamine uptake by this means was confined to the distal portion of the root and did not extend into the spinal cord.

\subsection{Force field analysis}

1.5.1 Force responses. Prior to the application of stimuli, we recorded $\mathrm{x}$ and $\mathrm{y}$ force components by placing the limb in a variety of locations. The resting forces we recorded varied according to the configuration of the limb. These variations were due to changes in muscle lengths, changes in muscle moment arms, and changes in the afferent feedback that modulates tonic postural activity. Following the microstimulation of the spinal cord, we observed a change in the forces at the ankle after a latency of between 30 and $150 \mathrm{msec}$. (Most response latencies were at about $100 \mathrm{msec}$.) The forces rose to a plateau level, and then declined to the baseline level at a variable period $(300 \mathrm{msec}$ to $2 \mathrm{sec})$ after the termination of stimulation. Each site in the cord produced a specific and repeatable balance of force components $\left(F_{x} F_{y}\right)$ at a particular workspace location (see Results, section 2.1; Table 1). Various criteria allowed us to distinguish direct stimulation of MNs from indirect activation involving synaptic integration. We judged records not to be direct MN activation when we observed the occurrence of one or all of the following three conditions: (1) a long (50-150 msec) latency of electromyogram activity, (2) electomyogram activity not time-locked to stimulus pulses, and (3) the persistence of electromyogram activity well beyond the stimulus train.

1.5.2 Active and resting forces. At each ankle location, the net force vector, $F$, obtained in response to a stimulation was expressed as the sum of two components - the "resting" force vector, $F_{r}$, and the "active" force vector, $F_{a}$ :

$$
F=F_{r}+F_{a} .
$$

The resting force vector corresponded to the force measured before the onset of the stimulus. The active force vector, $F_{a}=F-F_{r}$, represented the additional force induced by our stimulus. This induced force was different at each ankle location in the work space. It is likely that these two components, $F_{r}$ and $F_{a}$, result from the actions of different collections of muscle fibers and neural mechanisms. The resting force field was determined by the resting mechanical properties of the soft tissues and of all the muscles acting on the limb. In contrast, the active field was generated only by those muscles directly or indirectly activated in response to stimulation.
1.5.3 Measurement procedure. The major goal of our experiments was to determine the field of static forces associated with the stimulation of a spinal cord site. To examine the field, we measured the mechanical response at different ankle locations in the work space following the same spinal stimulation. Typically, we recorded the force vectors in a set of from 9 to 16 locations that formed a regular grid $(3 \times 3$ or $4 \times$ 4) over the limb's work space. [Here the term "work space" is used to indicate the range of movement of the ankle in the horizontal $(x-y)$ plane. The test grid in which forces were collected comprises only a portion of the total ankle work space.] At each grid location, we recorded the force vector elicited by the stimulation of a single spinal cord locus.

1.5.4 Force field reconstruction. By definition, a force field is a function relating every work space location to a corresponding force vector. For a single stimulation site, the force vectors measured at the different locations of the ankle in the work space were considered to be samples of a continuous force field. We used the measured force vectors to estimate the force field across a broad convex region of the ankle's work space. To this end, we implemented a piecewise, linear interpolation procedure according to the Delaunay triangulation algorithm (Preparata and Shamos, 1985). This algorithm partitions the work space into triangles as close to equilateral as possible. The vertices of each triangle were the tested grid points. In a piecewise, linear interpolation, the partition of the domain into equilateral triangles has the effect of smoothing the interpolating function. This partitioning also acts as a method for minimizing the average distance between the spatial locations of the measured data at each triangle's vertices and the points of interpolation in the interior of each triangle over the field.

Within each triangle, we applied a linear interpolation to the force vectors measured at the corners. Thus, within each interpolation triangle, the force components were given as

$$
\begin{aligned}
& F_{x}=a_{1,1} x+a_{1,2} y+a_{1,3}, \\
& F_{y}=a_{2,1} x+a_{2,2} y+a_{2,3} .
\end{aligned}
$$

Note that the above expression has six unknown parameters, $a_{i, j}$. Therefore, the interpolation problem with three data vectors (i.e., six data components) has a unique solution. It thus follows that a force vector at any point in the entire convex sampled space could be estimated from the interpolation parameters associated with the tessellation triangle that contained the point. Our interpolation procedure is an extension of the univariate interpolation of one-dimensional data. In a univariate piecewise linear interpolation, the data points are joined by a set of straight-line segments. Thus, the interpolating function is continuous but generally not smooth. In our bivariate interpolation, each component of the measured force vectors depended upon the $\mathrm{x}$ - and $y$-coordinates of the measuring site. The piecewise linear procedure corresponds to approximating each component's surface, $F_{x}(x, y)$ and $F_{y}(x, y)$, with a set of planar triangular patches. As in the univariate case, the interpolating functions are continuous (two neighboring triangles have two vertices in common) but not smooth (the interpolating parameters may change abruptly from one triangle to the next). However, unlike in the univariate case, the transition between two sets of linear coefficients does not occur at a single point but across the whole boundary segment between contiguous triangles. The purpose of the Delaunay triangulation algorithm is to keep the measure of this boundary at a minimum, that is, to give the interpolating surface the smoothest appearance compatible with a piecewise linear reconstruction and with the spatial distribution of the sampling sites.

The above interpolation procedure could be applied to the vector data collected at any given latency from the onset of the stimulus. Using data sets obtained at different latencies, we thus derived estimates of the evolution of a force field generated by a single spinal stimulation at differcnt sampling intervals. By examining a variety of different sample grids at different spatial densities, we determined a grid design that was adequate to capture the fields' variations. We then routinely used this design.

By definition, an equilibrium point is a location in the leg's work space at which both components of the force vector vanish. Thus, the presence of one or more equilibria was tested for by searching within each interpolation triangle for a location $\left(x_{0}, y_{0}\right)$ at which $F_{x}$ and $F_{y}$ were both zero. The accuracy of our estimate of the equilibrium point was tested as follows. After having identified an equilibrium point by our interpolation procedure, we measured three force vectors at the vertices of a small equilateral triangle $(1 \mathrm{~cm}$ side) centered at the estimated equilibrium site. Then, using only these three force vectors, we repeated 
the estimate of the equilibrium point within this smaller triangle. We found that the two estimates of the equilibrium point differed by less than $5 \mathrm{~mm}$. Considering that the average length of our frogs' legs was about $12 \mathrm{~cm}$ (from hip to ankle, with the leg fully extended), the discrepancy between the two estimates corresponds to an accuracy of $4.2 \%$. In several cases, we also explicitly confirmed that the actual measurements of forces were not significantly different from zero at the interpolated equilibrium position.

1.5.5 Active and resting fields. The field-reconstruction algorithm was applied to the resting force vectors, $F_{r}$, to the active force vectors, $F_{a}$ and to the net force vectors, $F=F_{r}+F_{a}$. We call the corresponding fields the "resting field," the "active field," and the "total field," respectively. We want to stress that the presence of an equilibrium point in the total field did not imply the presence of an equilibrium point in the active field. In these experiments, the presence of an equilibrium point in the active field was, however, a sufficient condition for an equilibrium in the total field. In many instances, the active field did display an equilibrium point. In this case, the equilibrium point of the total field moved toward the equilibrium point of the active field as the active forces increased in amplitude.

1.5.6 Field comparison and classification. In order to compare fields, we developed a collection of different tests that captured different aspects of force-field structure. We examined the fields using a number of statistics. Our data were the patterns of directly measured force vectors and the locations of equilibria. Before comparison, we normalized active fields. The active fields were normalized by finding the average magnitude of sampled vectors and scaling this average to unity. Thus, an individual vector was scaled:

$$
v_{s}=\frac{n v_{\mathrm{us}}}{\sum_{i=0}^{n} \sqrt{v_{i} \cdot v_{i}}}
$$

where $v_{\mathrm{s}}$ is scaled data vector, $v_{\mathrm{us}}$ is unscaled data vector, and $n$ is number of data vectors $\left(v_{i}\right)$ in the field. The reason for normalizing active fields in order to compare them arises from the observation that in many instances the active fields had very similar structure although their magnitudes varied. The magnitude at a given location was a function of stimulation strength, and the state and muscular strength of the preparation. While collecting fields, we made no explicit attempt to adjust magnitude to some specific level. However, the subsequent normalization allowed us to compare fields collected in different frogs. We routinely compared the pattern of the fields using the average of a weighted difference between the magnitude and the angular deviation of the vectors:

$$
d_{a, b}=\sum_{i=0}^{n}\left[\operatorname{abs}\left\{\frac{\Theta_{i}}{\Pi}\left[v_{b, i}+v_{a, i}\right]\right\}+\operatorname{abs}\left\{\frac{v_{b, i}-v_{a, i}}{v_{a, i}+v_{b, i}}\right\}\right],
$$

where $d_{a, b}$ is the measure of dissimilarity between fields $a$ and $b, \theta_{i}$ is the angle between the orientation of the $i$ th two force data vectors in radians, and $v_{a, i}$ and $v_{b, i}$ are the magnitudes of the $i$ th force data vectors in ficlds $a$ and $b$. The main purpose of this criterion was to detect qualitative differences such as the direction or gradient of the fields, as opposed to differences such as uniform scaling of the force amplitude across the work space. The rationale for this test criterion was that normalized sample force vectors drawn from fields could vary in two major ways. First, the orientations of vectors could differ, and second, the pattern of magnitudes could differ. The second type of variation could occur in a field in which the individual sample vectors' orientations did not differ. Finally, according significance to variations of either of these types depended on vector magnitudes. Thus, large angular differences derived from small vectors (close to the noise of our sensor) should not be accorded a great deal of significance. Hence, the orientation term $\left(\theta_{i}\right)$ was weighted by the magnitude of the vectors determining it. The second term in the equation measures the variation of magnitude of vectors. The divisor of this term accords magnitude variations across the field equal significance, regardless of vector size. While this makes the measure somewhat sensitive to noise in small-magnitude vector pairs, the divisor also prevents large-Inagnitude vectors from dominating the measure.

We also compared the equilibria obtained from the normalized active fields at the time of their peaks. The limb's work space was scaled to a constant area. This processing was applied to field samples collected on a standard grid, which was scaled with the frog's body length. The standard grid was composed of known joint angles at each site in the work space. The normalized active field was added to a uniform, con- stant-stiffness isotropic field with its equilibrium in the center of the work space. We then examined the locations and directions of the equilibria from the center of this scaled total field. In this way, we eliminated effects of varying stimulus strength, frog body size, and frog muscle mass on the comparison of field and active equilibrium point locations. These equilibria are a summary of the mechanical effects of the different types of active fields.

Each measure described above was used as a metric to compare any two fields. We used these measures routinely both in an exploratory cluster analysis and in the comparison of pairs of active fields. The purpose of the cluster analysis was to examine the variety and degree of clumping of fields into categories and thus to test the hypothesis that fields fall into a small number of separate classes. We performed cluster analysis on a data set of 49 fields gathered from five frogs. We used the nearest neighbor, the farthest neighbor, and an unweighted pair-group method, using arithmetic averages (Farris, 1969, 1973; Gordon, 1981). Clusters were chosen based on the separating interval or "moat" (see Gordon, 1981), that is, the size of change in the dissimilarity measured necessary to aggregate clusters. For example, after a level of 0.5 was reached, fields were aggregated in a few clusters on the basis of nearest neighbor analysis. These clusters could only be fused by accepting a far larger dissimilarity measure. For nearest neighbor analysis a level of 0.8 was required, that is, a "moat" of 0.3 , before any further aggregation occurred. These methods allowed us to develop a criterion for similarity of fields for test 4 : fields with $d<0.5$ were essentially similar and would almost always be in the same cluster.

1.5.7 Coordinate systems. Forces measured in Cartesian coordinates were converted to (1) torques in joint coordinates and (2) radial and tangential forces in polar coordinates centered on the hip. We performed these transformations of the data in order to examine in what coordinate frame the force fields demonstrated the simplest structure. These conversions were performed using estimates of the appropriate limb Jacobian based on measured kinematic parameters for each frog. The torque was then obtained by standard methods (Goldstein, 1981; Asada and Slotine, 1986).

\subsection{Measurement of muscle fields}

We compared the force fields elicited by the stimulation of the premotor layers of the spinal gray, with the force fields that could be expected from the independent activation of several leg muscles in the absence of feedback. The purpose of this comparison was to establish to what degree our findings could be accounted for by (1) unspecitic random muscle recruitment through current spread in fiber systems and in motoneuron's dendritic trees and (2) specific recruitment based on the known motoneuron topography (Cruce, 1974b). To this end, we built a data base of force fields elicited by direct muscle stimulations in deafferented animals. We used this data base to predict the force field corresponding to several combinations of muscles as described in the Appendix.

Muscle fields were measured as follows. Our force-measuring instrument was attached firmly to the frog's calf just above the ankle joint. Our attachment constrained the limb's nominal end-point to a horizontal plane. The pelvis was firmly clamped and immobilized. In this arrangement, the configuration of the limb (the hip and knee angles) at any work space position was, in principle, fully constrained proximal to the ankle: there were no excess degrees of freedom. As a result, the isometric actions of individual muscles generating end-point forces could be examined in isolation. A muscle's force field was obtained by recording the $\mathrm{x}$ and $\mathrm{y}$ forces at the ankle. A force was produced at each location of the tested work space when we electrically stimulated each muscle through the implanted electrodes. We measured the force fields generated at the ankle after direct stimulation of each of 12 muscles that together constituted $90 \%$ off the leg's muscle mass.

\section{Results}

It is well known that spinalized frogs can generate limb trajectories aimed at parts of the body after the application of noxious stimuli to the skin. However, we have discovered that repeatable and well-organized motor responses can also be evoked by microstimulating different portions of the lumbar spinal cord. We found that the electrical stimulation of the dorsal and lateral 


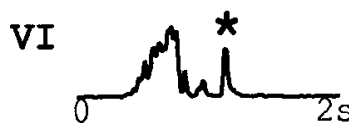

ST

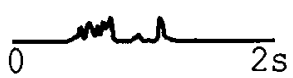

SM

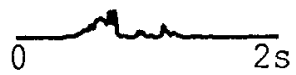

$\mathrm{AD}$

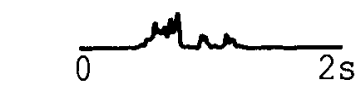

RI

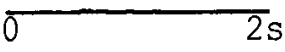

RA

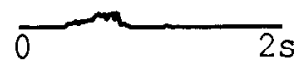

GA

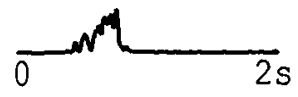

TA

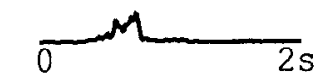

VE

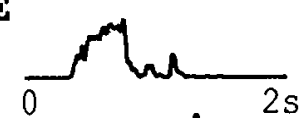

SA

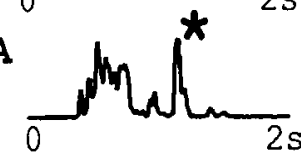

BI

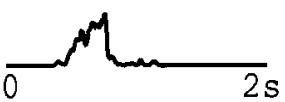

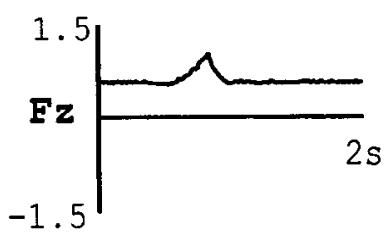
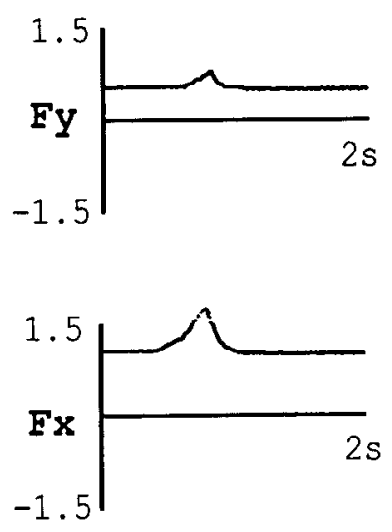

Figure 2. Sample data collected at one point in the collection grid for a spinal cord stimulation in the spinal gray. Left and middle columns, Rectified and integrated EMG and stimulus (solid trace). $V I$, vastus externus; $S T$, semitendinosus; $S M$, semimembranosus; $A D$, adductor magnus; $R I$, rectus internus; $R A$, rectus anticus; $G A$, gastrocnemius; $T A$, tibialis anticus; $V E$, vastus externus; $S A$, sartorius; $B I$, biceps. Right column Force traces: $F x, F y, F z$ translational force components (in newtons). Notice the latency of EMG and the rebound activations (marked with asterisks) following cessation of stimuli. Forces consist of a steady offset (resting force) and a response to stimulation. The response here is transient, but plateau and transient/plateau responses were also seen (see Table 1, Fig. 3). Stimulation consisted of a $600 \mathrm{msec}$ train of $6 \mu \mathrm{A}$ pulses at a rate of $50 \mathrm{~Hz}$. The trace shown formed a component sample for the fields in Figures 5-7. neuropil regions of the spinal cord, at threshold, resulted in the coactivation of a group of the leg's muscles (Fig. 2). We observed that different groups of leg muscles were activated as we moved the stimulating electrodes to different loci of the lumbar spinal cord in the rostrocaudal and mediolateral direction.

\subsection{Force and muscle-recruitment patterns: stability with depth}

As the electrode penetrated deeper into the cord, the orientation of the "active," above-baseline force vector recorded at a given leg position generally remained constant. Its magnitude usually varied, reaching a peak at the site of lowest threshold. Normally, as long as a single electrode penetration remained in the lateral neuropil, the orientation was maintained at depths ranging from 600 to $1100 \mu \mathrm{m}$, and the same collection of muscles was activated. An example is seen in Figure 3 . The average active force vectors are shown for all depths of a penetration. Notice that the vectors are of constant orientation over a broad range of the intermediate gray and that this orientation of the forces differs from that found in the deeper MN pools. We interpreted this result to mean that a constant balance of muscle responses

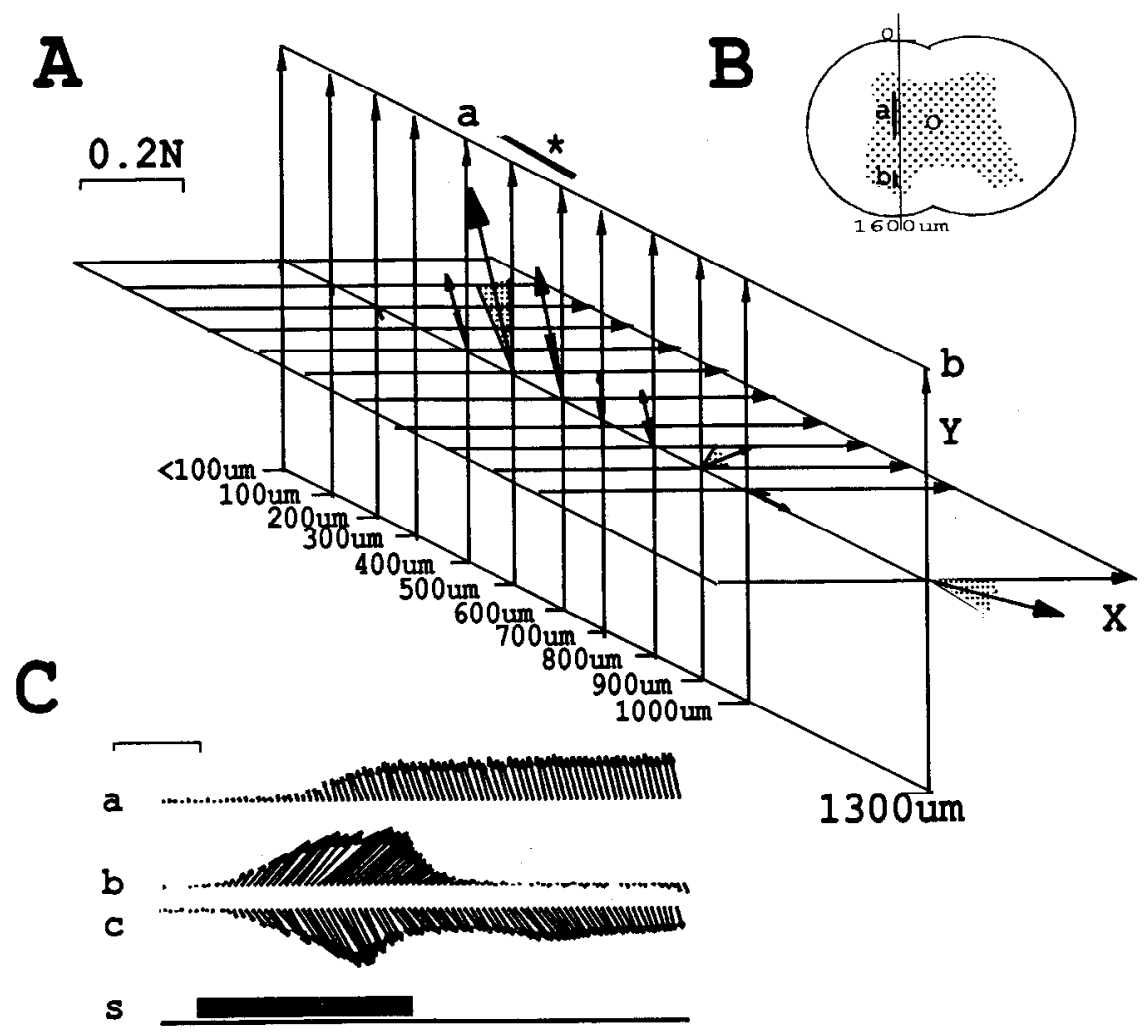

Figure 3. The orientation of average force with depth. $A$, The orientation of the average force vector at successive depths in the spinal cord. The force vector displayed is composed of components $F x$ and $F y$. The baseline forces were removed. These force vectors were elicited by a $2.7 \mu \mathrm{A}, 60 \mathrm{~Hz}, 300$ minsec train. The shaded triangles are SDs of the individual force vectors. Notice the constant orientation of the vectors for the $500 \mu \mathrm{m}$ depth starting at a. When the electrode penetrated deeper (to b) among the motoneuron somas, a different orientation was found. $B$, Schematic cross section of cord showing electrode track locations. $C$, Growth over time of the vectors at spinal cord sites. Calibration, $0.2 \mathrm{~N}$. The force vector typically showed one of three behaviors shown here. The top trace is the force actually recorded at point a in $A$. $a$, the most common response is a smooth rise to a force plateau; $b$, a typical transient response; $c$, a typical transient/plateau response. 


\begin{tabular}{lcc}
\hline Table 1. Force responses at individual sites \\
\multicolumn{4}{c}{} \\
Classification of response types at each stimulation location \\
Plateau response & 254 & $37 \%$ \\
Transient response & 165 & $24 \%$ \\
Transient/plateau & 198 & $29 \%$ \\
Multiphasic & 32 & $5 \%$ \\
Unclassified/silent & 33 & $5 \%$ \\
Variance of active force orientation over time & \\
Variation $<12^{\circ}$ & 524 & $77 \%$ \\
Variation $12-17^{\circ}$ & 87 & $13 \%$ \\
Variation $>17^{\circ}$ & 71 & $10 \%$ \\
Stability within penetration: variation & $<12^{\circ}$ (118 penetrations) \\
Stable over $300 \mu \mathrm{m}$ & 54 & $49 \%$ \\
Not stable & 64 & $51 \%$ \\
Qualitative stability (same orientation in same quadrant) \\
Stable & 100 & $85 \%$ \\
Unstable & 18 & $15 \%$
\end{tabular}

Data are results of 682 stimulations in 118 penetrations ranging in depth from 400 to $1200 \mu \mathrm{m}$. Penetrations were placed in a grid across the lumbar cord in six frogs. The grid had two rows $200 \mu \mathrm{m}$ or $400 \mu \mathrm{m}$ from midline with rostrocaudal electrode separations of $1 \mathrm{~mm}$. Statistics are presented for active force variance and stability with depth.

was elicited throughout the central range of these depths of the penetration. Table 1 shows the types of force response and the range of variations of individual force vectors that were seen as the electrode penetrated the spinal gray in various locations in six frogs. With respect to the time course of the forces, Table 1 indicates that most responses fell into a few categories. Many force responses had the form of a plateau that outlasted the stimulus. More phasic, transient responses occurred in some instances, and combincd transicnt and plateau responses also occurred (see Table 1). In general, the orientation of the force in both transient and plateau in the combined responses was similar. With respect to the place of stimulation in the gray, the response usually remained qualitatively stable over depths from $400 \mu \mathrm{m}$ to $800 \mu \mathrm{m}$. By "qualitatively stable," we mean that the

\section{Table 2. Field convergence statistics}

Total fields

Convergent to equilibrium in tested area

Convergent to equilibrium not in tested area

85

$37 \quad 30 \%$

Active fields

Convergent fields

Parallel fields

Divergent fields

Equilibrium locations for active convergent fields In tested area

Outside tested area

$\begin{array}{rr}101 & 83 \% \\ 20 & 16 \% \\ 1 & 1 \% \\ & \\ 15 & 12 \% \\ 107 & 88 \%\end{array}$

Data are results of an analysis of field patterns and equilibrium locations in a sample of 122 fields collected in 24 frogs.

elicited forces remained within the same quadrant of space; $50 \%$ were stable by a stringent quantitative criterion. In a few stimulations, a second phase of responses followed the first, usually at a latency of $500 \mathrm{msec}$. A few locations produced no responses.

Two considerations were important in our subsequent measurements of a force field. First, we made sure that the electrode was placed at a depth in the spinal cord that corresponded to the lowest force threshold. Second, in choosing a location for a force field, we included only those penetrations in which the force vector remained directionally invariant for at least 300 $\mu \mathrm{m}$.

\subsection{Convergent force fields}

To record the spatial variations of forces generated by the leg muscles, we followed a three-part procedurc. First, we placed the frog's leg at a location within the leg's work space. Second, we stimulated a site in the spinal cord and recorded the direction and amplitude of the elicited isometric force at the ankle. Third, we repeated the stimulation procedure with the ankle placed at each of 9-16 locations. These locations sampled a large portion
Figure 4. Resting fields and the separation of active and resting fields. Top, Three different resting fields that were collected from a single frog over $6 \mathrm{hr}$. These have an almost constant structure. Bottom, An example of the removal of the resting field (measured at $200 \mathrm{msec}$ prior to stimulation) from the total peak field measured at $450 \mathrm{msec}$ (250 msec following stimulation). The vector-by-vector subtraction used to obtain the active data is equivalent to the removal of baseline forces. The removal generates an "active field." It can be seen that in this case the active field was convergent. Parameters: $9 \mu \mathrm{A}$ at 60 $\mathrm{Hz}$ for $300 \mathrm{msec}$ at $400 \mu \mathrm{m}$ depth in cord.
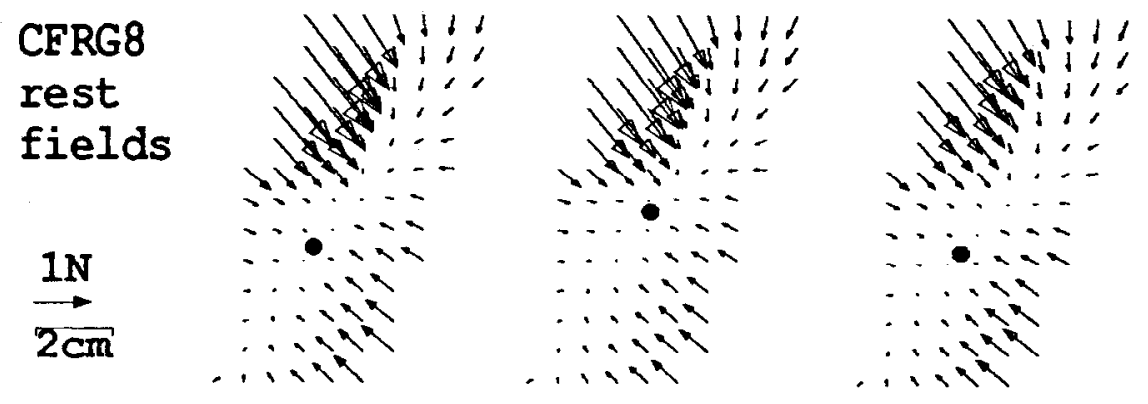

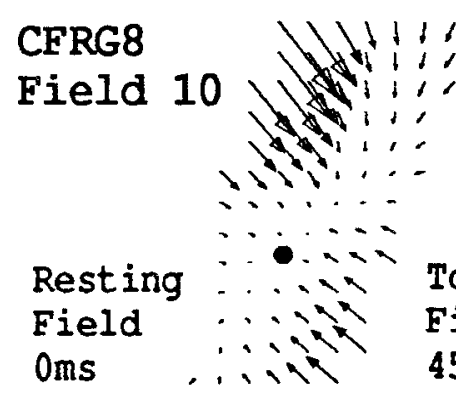

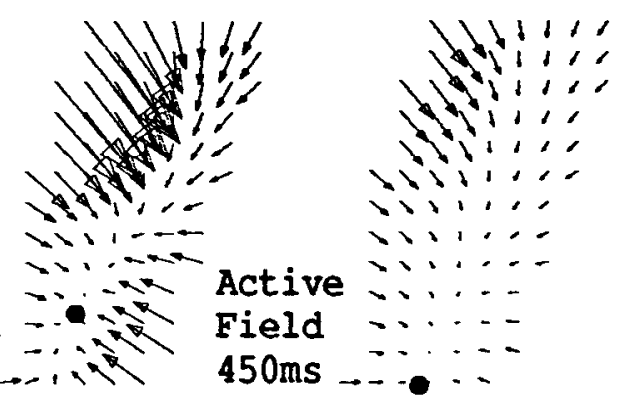


$200 \mathrm{~ms}$

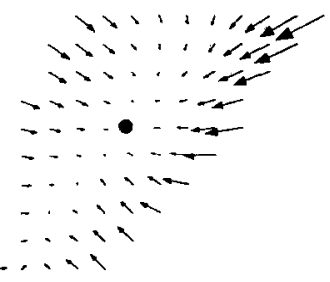

$350 \mathrm{~ms}$

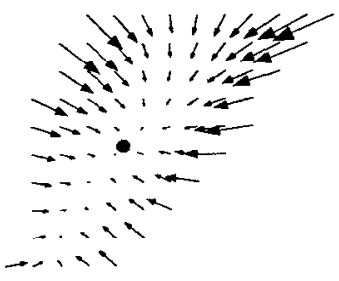

$500 \mathrm{~ms}$

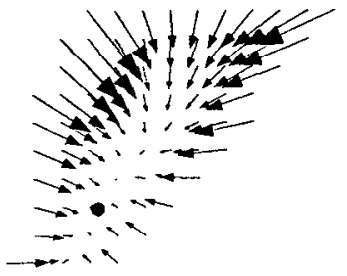

$250 \mathrm{~ms}$

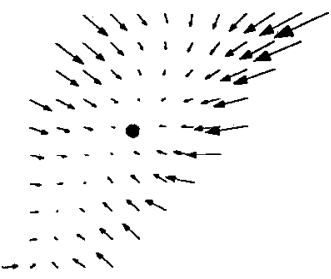

$400 \mathrm{~ms}$

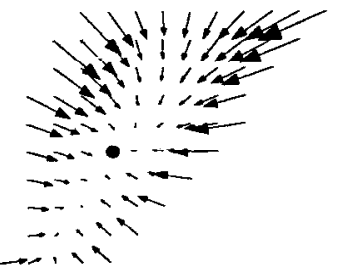

$550 \mathrm{~ms}$

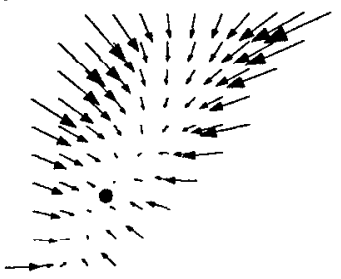

$300 \mathrm{~ms}$

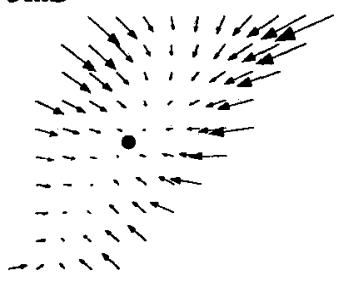

Virtual Trajectory

$450 \mathrm{~ms}$

$600 \mathrm{~ms}$
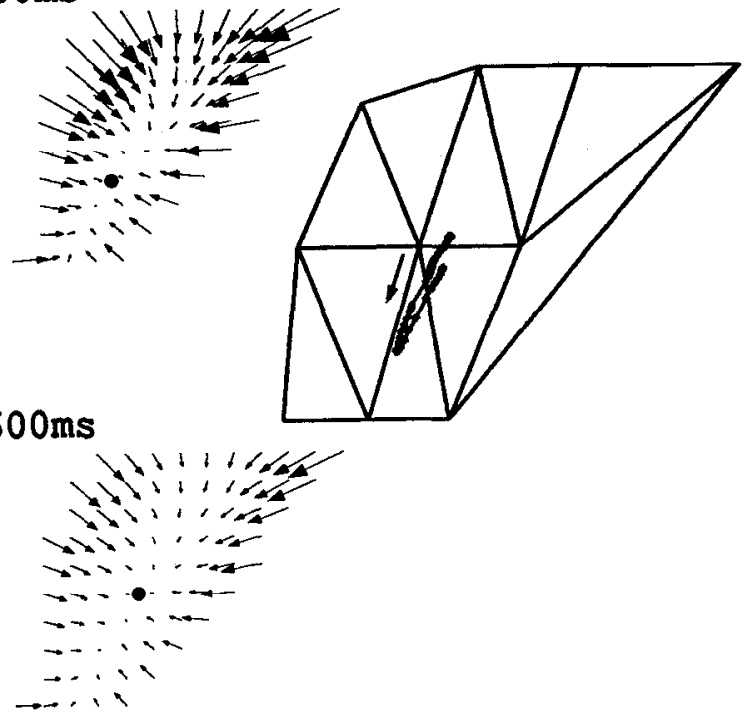

Figure 5. Temporal evolution of the total force field. The total force field specifies the mechanical equilibrium of the limb. Over time, the field stiffens and alters as a result of microstimulation. The equilibrium point of each total field is shown. The motion of this equilibrium forms the virtual trajectory, which is shown to the right. Stimulation parameters are $1.5 \mu \mathrm{A}$ and 0.6 -msec-duration pulses at $40 \mathrm{~Hz}$ for $300 \mathrm{msec}$. The time shown in the upper right of each field was measured relative to the stimulus train onsct. Comparc the pattern of motion of the equilibrium in the figure with the active field behavior shown in Figure 6 for the same field.

of the region of the horizontal plane that could be reached by the frog's ankle (i.e., the leg's work space). At each location, we stimulated the same site in the spinal cord and recorded the force vector, $F=(F x, F y)$. Although the site of spinal cord stimulation was constant throughout, we found that the elicited force vector varied as we placed the leg at different locations. These changes in force through the work space resulted from a variety of kinematic and mechanical factors such as the length, the moment arms, and the viscoelastic properties of the muscles. In addition, reflex modulation of the muscles' activations played a role in the development of the forces at the ankle.

The collection of the measured forces corresponded to a force field (Figs. 1, 4; see also sections 1.5.1-1.5.5). Remarkably, in most instances (Table 2; see also section 2.5 ), the spatial variation of the measured force vectors resulted in a field that was at all times both convergent and characterized by a single equilibrium point (i.e., a point at which the amplitude of the $F x$ and $F y$ force components was zero). This equilibrium point represented the locus at which the leg would have been at steady state if it were free to move (see also section 2.10). That is, the equilibrium point was the position at which there would be no end-point force. We will refer to a field with this convergent pattern as a convergent force field (CFF).

\subsection{Virtual trajectory}

The temporal evolution of the total force field caused a smooth movement of the equilibrium point from its position at rest to a new position at peak force magnitude and then back to the resting position. This motion of the equilibrium point constitutes what has been called the "virtual trajectory" (Fig. 5; Hogan, 1985). (The virtual trajectory shown in Fig. 5 may bear some resemblance to the kinematics observed in the earliest phase of wiping to the back or forelimb.) The virtual trajectory is defined as the trajectory of positions at which the leg would experience no net forces. More significantly, this is also the trajectory of spatial locations toward which the limb end-point would be attracted at any instant in time if it were unconstrained by our apparatus. The movement of the equilibrium could hence be used by the CNS to generate postural transitions and movements (Hogan, 1984, 1985). We will show in a subsequent section that this is indeed the case.

\subsection{Stability of resting fields}

The prestimulus resting field of our preparations remained stable over each day and often over several days of recording, as long as the preparations were reflexive. This field represents a 

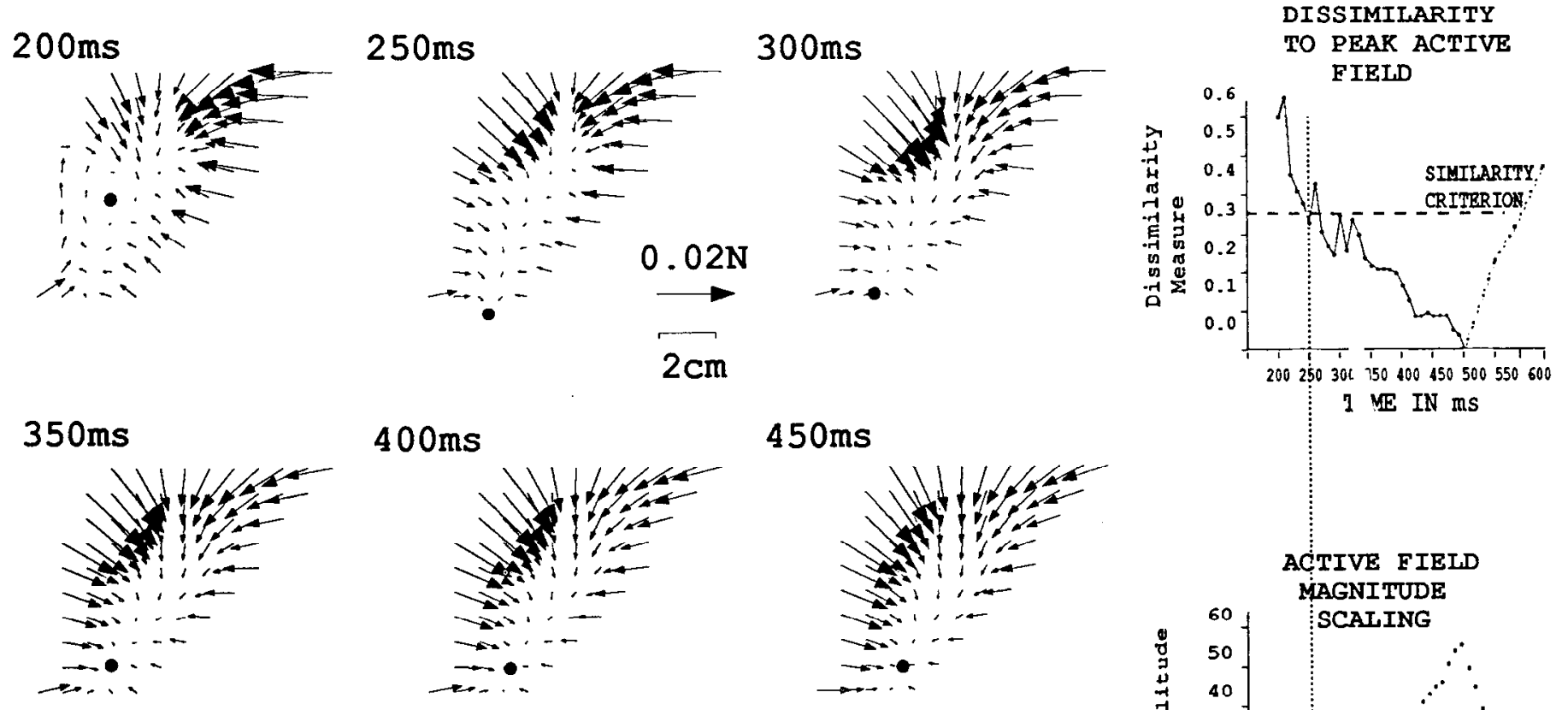

$500 \mathrm{~ms}$

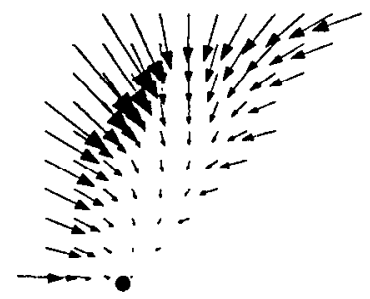

$550 \mathrm{~ms}$

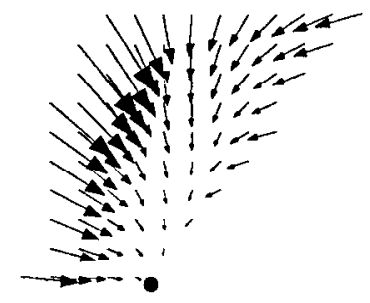

$600 \mathrm{~ms}$

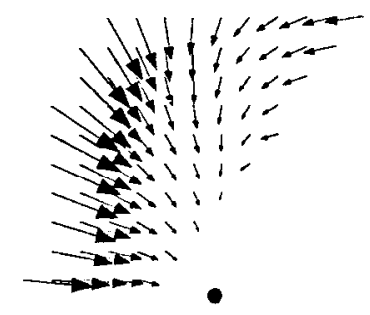

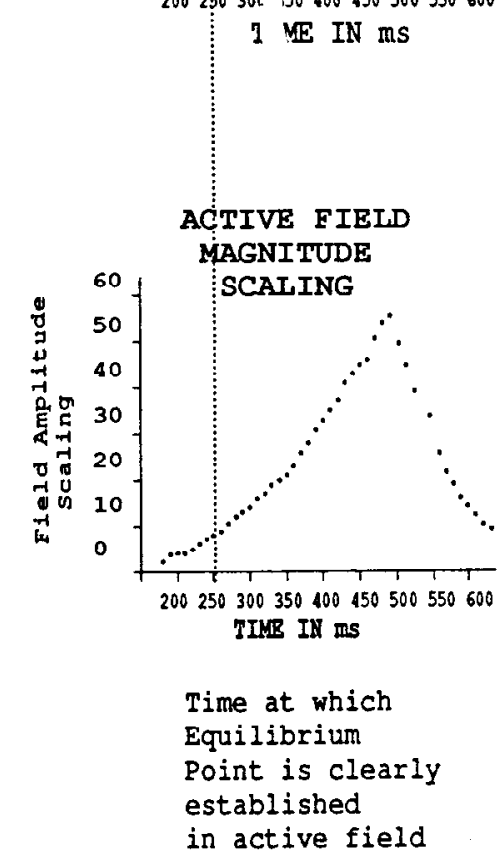

Figure 6. Normalized active fields are almost constant. To obtain this figure, active fields were obtained by removing the resting field from the fields shown in Figure 5. These active fields were then scaled to a constant average value to produce a normalized active field (Eq. 3). The normalized active fields are shown at the left. The magnitude of the scalar by which each of the normalized fields would have to be multliplied in order to obtain the original active field magnitude is shown in the graph to the lower right. Notice that after about $250 \mathrm{msec}$, the normalized field had an almost constant structure until $600 \mathrm{msec}$ (when it begins to relax). A similarity measure for each active field relative to the peak active field was calculated and is in the upper right. After $250 \mathrm{msec}$, the similarity measure value dropped helow the criterion value. This means the fields are viewed as the same by this measure. The action of the microstimulation over time can be thus taken to be a scaling of the amplitude of a normalized active field of invariant structure. (Stimulation parameters are given in Fig. 5.)

combination of resting postural mechanisms and passive biomechanics of the leg (Fig. 4). Although relatively constant within a single frog, these resting fields sometimes differed between individual frogs. For this reason, when comparing frogs, we subtracted the contribution of the resting fields from the total fields.

\subsection{Separation of fields into active and resting reduces variability in data}

We found that the interpretation of the force field data was simplified when the baseline forces (representing the resting field) were removed. To this end, we implemented the following procedure. We divided the total field into a prestimulus resting field and a poststimulus active field. We performed this separation by means of a point-by-point vector subtraction of forces in the prestimulus resting field from the field that resulted from our stimulation. (This procedure is described in Materials and Methods. An example of this procedure is shown in Fig. 4.) The operation was thus equivalent to removing the baseline forces.
In part, the rationale for this procedure rests upon the fact that frog motor units are divided into two types: the slow-fiber system and the phasic-twitch fiber system.

Following a division of the total field into active and resting fields, we found there were important distinctions between the active and the total fields. The equilibria of the total recorded fields were spread across the work space. However, we found that our active-field data fell into a few classes or force field types.

\subsection{Time course of active field development}

We found that the active force fields following stimulation were usually convergent $(\sim 80 \%)$, although there were exceptions as noted below (see Table 2). Following the decomposition of a force field into active and resting fields, we found that (1) the amplitude and stiffness of the active field rose and fell while (2) the directions and balances of the pattern of forces in the field were invariant as the pattern evolved in time. The force pattern at any instant of time was a scaled version of that seen at the 


\section{6uA 300ms}

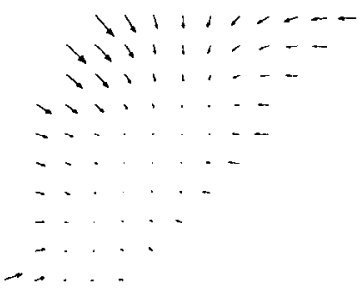

$7 \mathrm{uA} 450 \mathrm{~ms}$

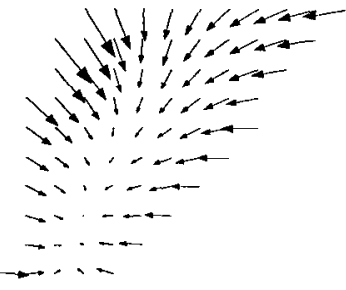

$12 \mathrm{uA} 450 \mathrm{~ms}$
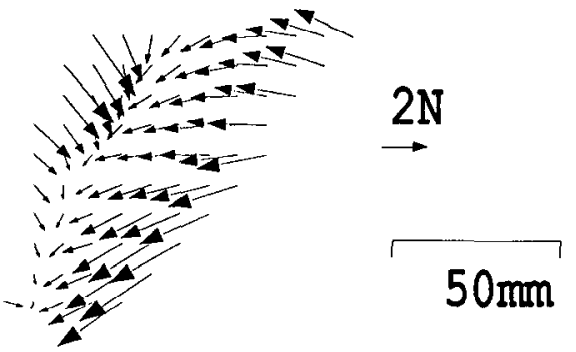

6uA $450 \mathrm{~ms}$

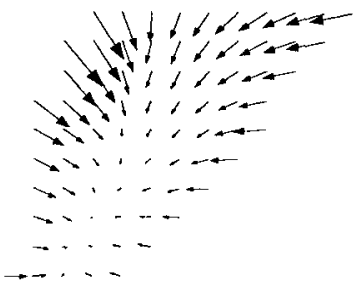

8uA 450ms

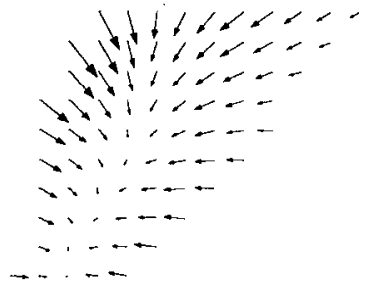

\section{6uA 600ms}

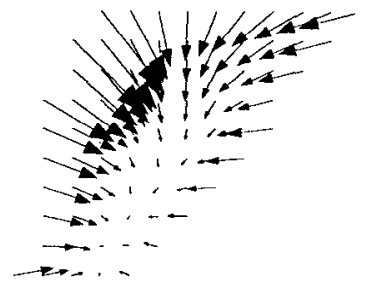

\section{9uA 450ms}

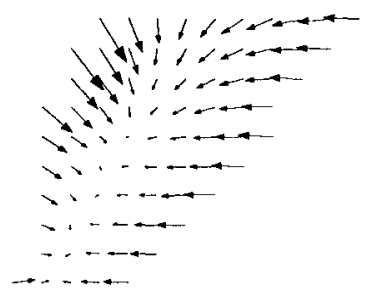

Figure 7. Modulation of active-field strength by stimulus parameters. A collection of peak (unscaled) active fields is shown. These were obtained at a single spinal cord site in the intermediate gray at different settings of stimulus parameters. The electrode locus in the cord was the same as that at which the fields shown in Figures 5 and 6 were collected. Varying train duration strengthened the field, but this occurred without altering the field structure (top row). It can be seen that varying the current strength had only a small effect on peak amplitude and produced no change of field structure (middle row). Only at the highest current of $12 \mu \mathrm{A}$ did the field alter in structure and equilibrium point. The matrix of similarity measures among these fields is shown to the lower right. A measure that is less than 0.5 means that fields are almost identical by this measure. Fields' numbers are left to right and top to bottom. The matrix indicates that at $12 \mu \mathrm{A}$, field 7 differed significantly from field 2 . Fields $1-6$ were all similar. Field 7 would be separated from fields $1-6$ on this basis in a farthest neighbor cluster analysis. time of the peak magnitude of the fields. If present, the equilibrium of the active field remained at a fixed location through time. This force-pattern invariance can be seen clearly in Figure 6 . The force vectors of the active field varied in magnitude over time, but did not vary in direction or balance.

Active fields showed considerable stability both through time and in repeated trials. For example, in eight repetitions of a field obtained by stimulating the middle gray, the orientation and magnitude variations of the eight active fields were small (dissimilarity measure $d \ll 0.5$ ). The resting fields were also stable. As a result, the equilibrium of the total field at peak force had an SD of position of only $2 \mathrm{~mm}$ in $\mathrm{x}$-coordinate (SE, $0.8 \mathrm{~mm}$ ) and $0.5 \mathrm{~mm}$ in $\mathrm{y}$-coordinate $(\mathrm{SE}, 0.19 \mathrm{~mm}$ ). From the average orientation variations in Table 1 , the predicted equilibrium SD would in general be less than $6 \mathrm{~mm}$ for a grid of equilateral triangles with $2 \mathrm{~cm}$ sides. The observed variation is thus better than expected (see also section 1.5.4).

The active field had a fixed equilibrium point through time and in repeated trials as described above. However, the equilibrium of the total field smoothly followed a trajectory. This was achieved as follows. The total force field was determined by the vector sum of active and resting fields. Thus, at any point in time, the location of the equilibrium point was determined by the relative balance between the scaling of the active field and the resting field. In this way, the waxing and waning of the active field over time caused a smooth movement of the actual mechanical equilibrium of the limb to a new location and back again. When a plateau response was seen, the equilibrium point paused at the final location for a prolonged time. Note that, after a delay from the end of the stimulus, the equilibrium point returned to the equilibrium location of the resting field. Presumably, in natural movements, the new equilibrium point specifies a posture that is maintained until the animal decides to shift to a new location (by activating a second active field and relaxing the first) (see section 2.17 ).

The total ficld's virtual trajcctory is shown in Figure 5. The invariance of the active field's structure that underlies the total field's virtual trajectory is shown in Figure 6. Compare the scaling parameters for field magnitude and the stiffness of the active field over time in Figure 6 with the virtual trajectory and equilibrium positions seen in Figure 5 . It can be seen that the stiffness of the active field in Figure 6 increases to a peak value and then declines in synchrony with the movement of the equilibrium to its peak position and back again in Figure 5. The peak stiffness of the active field corresponds to the most extreme position of equilibrium movement. It is this active field magnitude and stiffness modulation that combines with the resting field to form the virtual trajectory.

\subsection{Exceptions to active field invariance}

The few instances in which we observed a change in the active force pattern over time occurred either at the lowest currents or shortly after stimulation. This change appears in regions of the work space where participating muscles were presumably slackened prior to stimulation. One possible cause for these force-orientation changes is thus an imbalance in the dynamics of muscle contractions at these locations. 

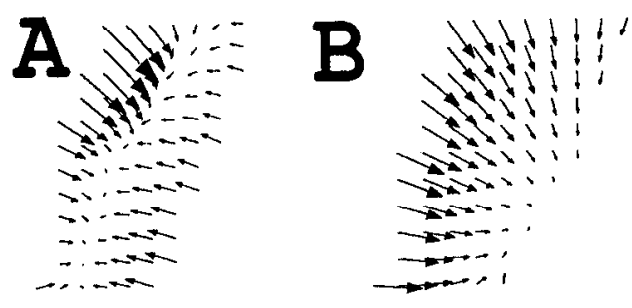

Figure 8. Sample fields drawn from a set of 49 fields at different points and in five different frogs. These 12 fields are examples of four typical force patterns obtained in the spinal gray. $A$, Rostral flexion (cluster A in Fig. $9 B$ ). $B$. Flexion withdrawal or hip flexion (cluster $\mathrm{B}$ in Fig. $9 B$ ). $C$, Caudal directed field. $D$, Extensor field (cluster $\mathrm{C}$ in Fig. 9B). Compare these fields with the data in Figure 9, which examines 49 of these field samples in detail to estimate the degree of clustcring into types. These fields were scaled to about the same magnitude. The pattern of forces in the figure, not the magnitude, are to be compared.
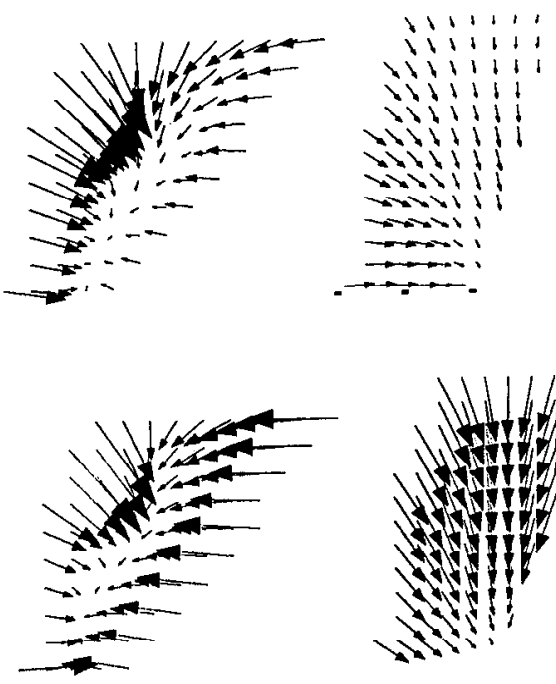

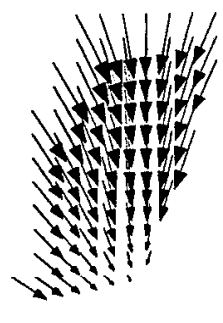

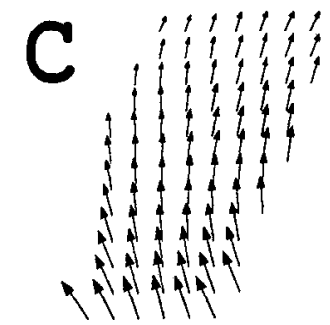

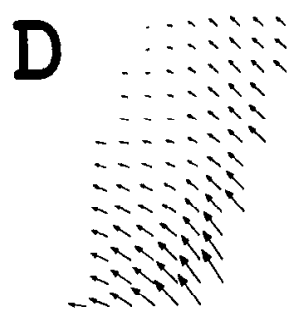

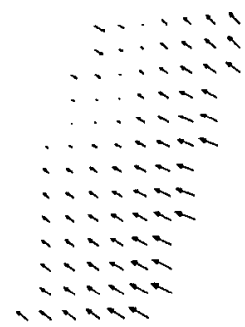
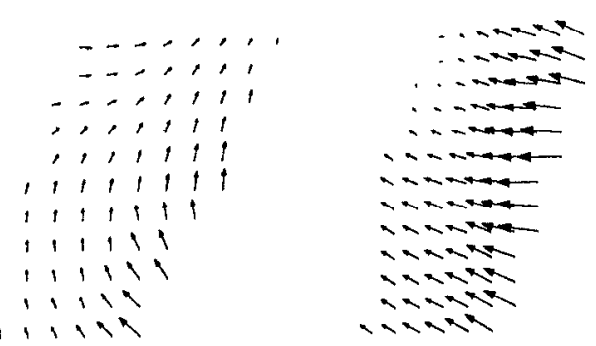

of these cells. That these two different methods of stimulation lcd to similar results suggests that there is some integration of activity underlying CFFs that can occur over time or as a result of activating a larger population. Given the range of more than $300 \mu \mathrm{m}$ over which a similar synergy and CFF were elicited, the similarity of these two effects is not unexpected.

\subsection{Similar fields are elicited at several sites in the spinal gray}

We observed that active fields at a variety of sites appeared to be similar in structure (Fig. 8). To confirm this impression, we computed similarity measures for a collection of such fields. (This procedure is described in Materials and Methods.) Each type of measure strongly supported the notion of the existence of a few clusters.

We also wished to compare the equilibria that would result from active fields under identical conditions. The active fields varied in magnitude depending on strength of stimulation, strength of the individual frogs musculoskeletal system, and the individual site that was stimulated. However, qualitatively there appeared to be great similarity among fields at different locations in different frogs. To examine the actual equilibrium positions that these fields would generate at similar strengths of response, we normalized fields to a standard magnitude. While fields were collected from frogs of different sizes, the sampling grids were designed to be directly comparable after spatial scaling. The fields were therefore also spatially scaled. Finally, we observed that resting fields could differ from frog to frog (see section 2.4). We therefore designed an artificial resting field that we used to replace the individual frogs' resting fields.

As a standard resting field, we chose an isotropic (radially symmetrical) convergent field with its equilibrium point in the center of the work space. The equilibria resulting from the summation of active fields with this standard field may be expressed 


\section{A: scaled equilibrium distributions}
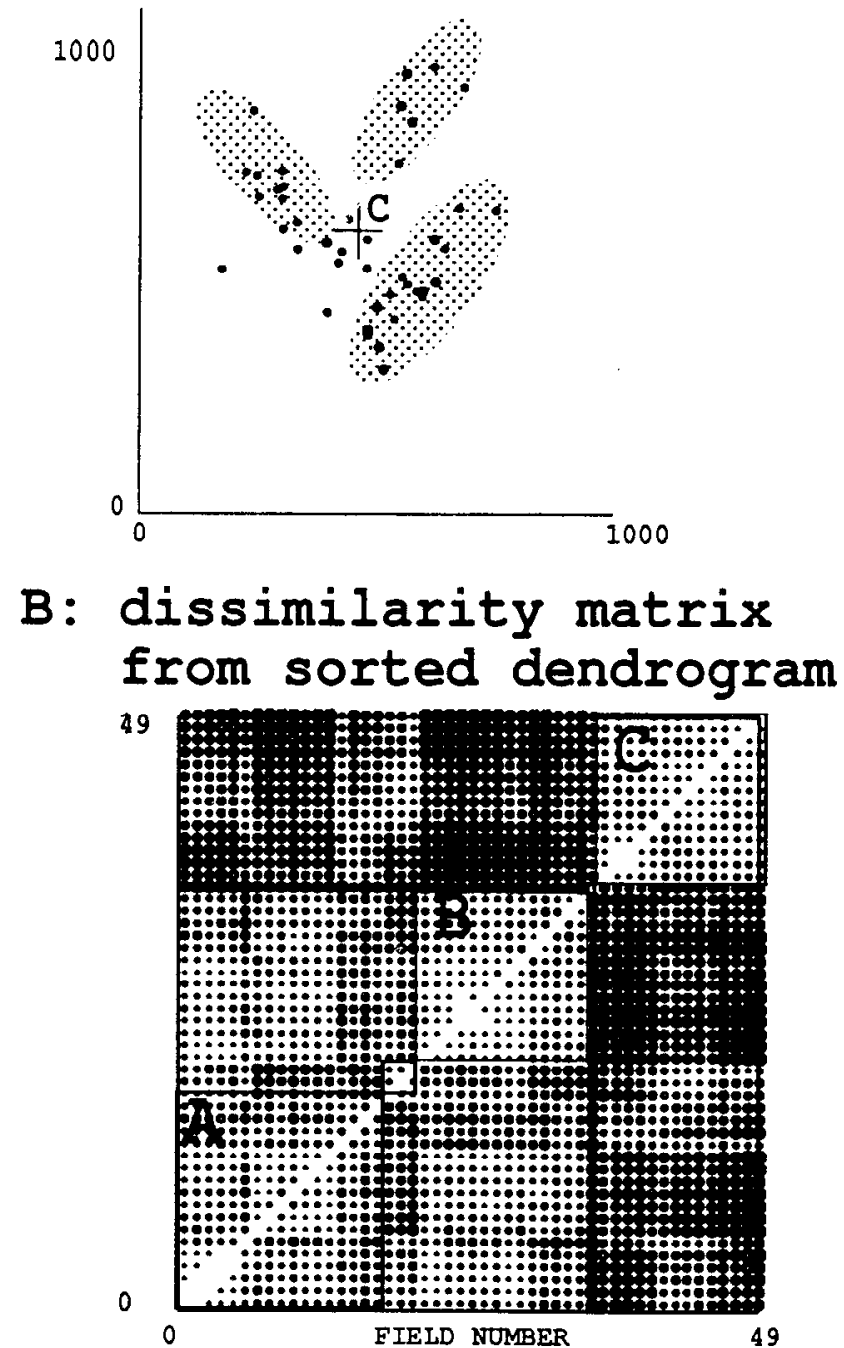

49

\section{C: scaled equilibrium directions}

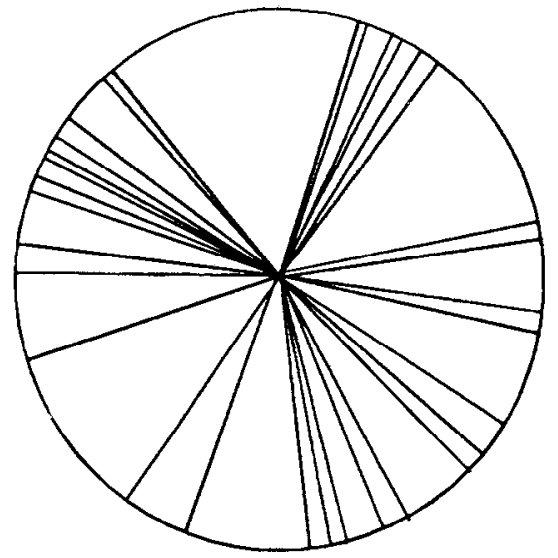

D: directions histogram

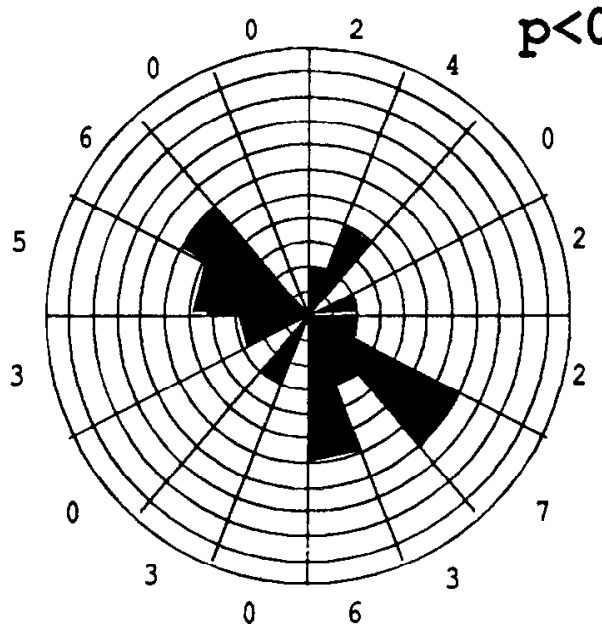

Figure 9. Clustering of fields into a few types. $A$, Distribution of equilibrium points. To obtain this distribution of equilibrium points, 43 normalized peak active fields from five different frogs were scaled in space so as to all occupy the same uniform work space (Eq. 3). These fields were then added to a standard radial isotropic field centered at $\mathbf{c}$. It can be seen that there appear to be a few groupings (indicated by shading). $B$, Dissimilarity matrix. A dissimilarity matrix of 49 normalized active fields from five different frogs is shown. The elements of the matrix show the dissimilarity between two fields (darker circles represent less similarity). The matrix is thus symmetrical and the diagonal elements are 0 . The dissimilarity measure used was based on a flow patterning measure (Eq. 4). The measure revealed several clusters. The panel shows the dissimilarity matrix after sorting the fields based on the farthest neighbor dendrogram. The orders of the elements were sorted so that fields in the same cluster were adjacent to one another in rows and columns. The lighter squares along the diagonal (outlined and marked $A-C$ ) therefore represent clusters (sample fields from each cluster are shown in Fig. $8 A-C$, respectively). The similarity among different clusters can be judged from the off-diagonal blocks of elements (e.g., elements in the block from $[35,20]$ to $[49,34]$ show that clusters $B$ and $C$ resemble one another very little. $\Lambda$ ny two fields with a dissimilarity measure of less than 0.5 between them could be classified as belonging in a single cluster; see Materials and Methods). $C$, Equilibrium directions. A plot showing the individual directions of the movements of the equilibria of the fields represented in $A$. The movements are all with reference to the location c. $D$, Dircction histogram. The distribution of direction vectors shown in $C$ is displayed as a circular histogram of 16 sectors, showing the number of equilibria in each sector. The histogram has five sectors that are completely empty. The chance of there being five empty sectors for randomly distributed equilibria is less than 1 in $1000(p<0.001)$. The statistics of this representation of our data therefore support the hypothesis that there was a clumping of force fields into a few types with characteristic directions.

as directions away from the central equilibrium of the isotropic resting field. Viewed together, the equilibria's displacements from the center can be seen to cluster about a few principal directions. The similar directions of movement of these fields at different spinal cord sites suggest that distant areas of the cord are similar with regard to the synergy and force fields generated. Figure 9 shows the results of this analysis based on the equilibria found when normalized active fields were added to a standard resting field.
We confirmed this result using a second scheme based on the classification of directions of vectors in field patterns using cluster analysis. (This procedure is described in Materials and Methods.) We found that within our sample of 49 fields from five frogs, the data fell into a few classes. Examples of three fields from each of four classes derived from several different frogs are shown in Figure 8. The two analyses shown in Figure 9 reveal only a few field types. (This catalog of fields is currently being pursued in more detail.) 


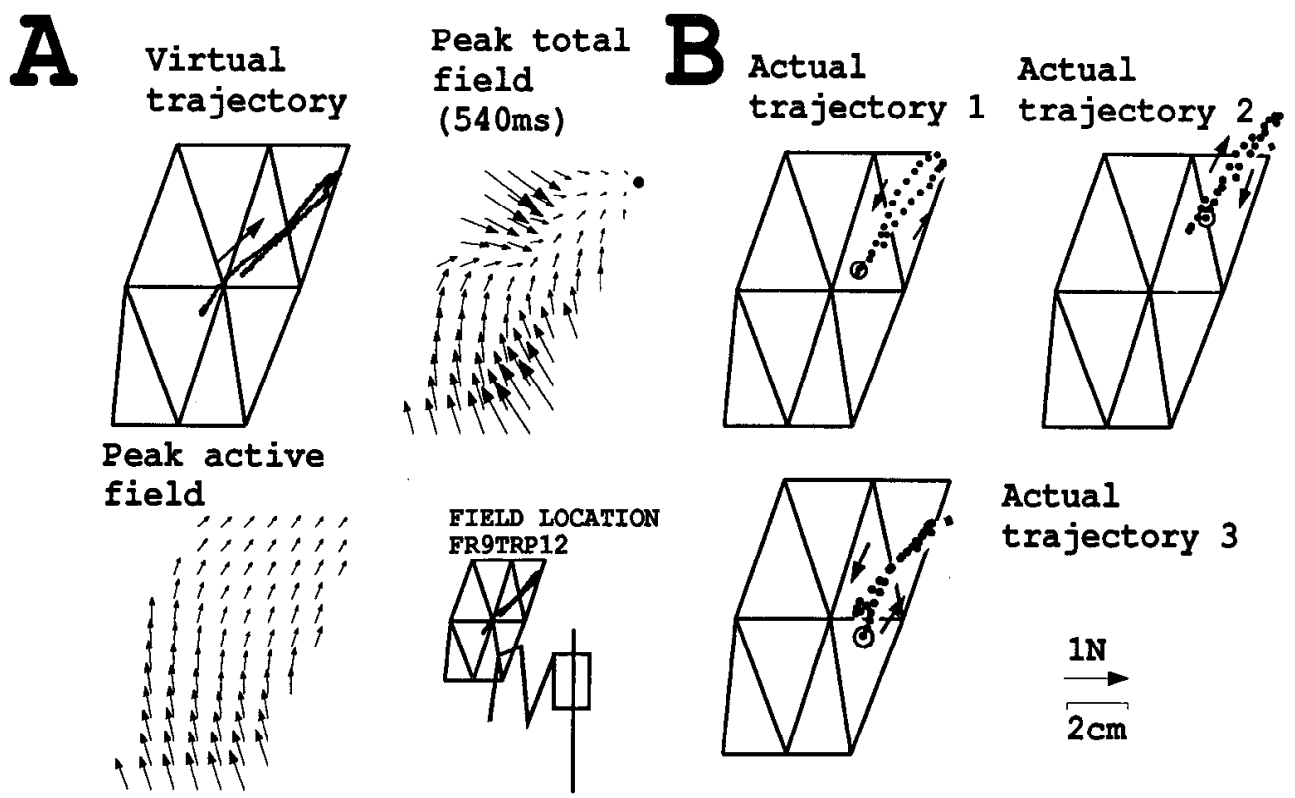

Figure 10. Comparison of actual and virtual trajectory. A, Force field measurements. The virtual trajectory of equilibrium points is shown (upper left). The virtual trajectory derives from the time course of the total convergent force field shown at peak amplitude. The active field (lower left) had a pattern of forces that would specify a flow moving caudally and medially. The relation of the trajectory to the frog's body is shown schematically at lower right. B, Trajectory measurements. Three sample limb trajectories from the suspended limb are shown. These were recorded at $30 \mathrm{~Hz}$ using a video system. We related the kinematic data to the grid of points used to collect the forces sampled for the field. The three trajectories begin at different locations in the grid. This was due to different initial resting fields as a result of the leg's suspension (see text). The actual pattern of movement is well predicted by the total field. This was especially evident in the first trajectory where resting equilibria almost coincide. The direction of the movement was well predicted by the active pattern in those trajectories where initial resting postures differed.

\subsection{Movements induced by microstimulation compared to field virtual trajectories}

In a preceding section, we showed that we could divide our measured total CFF into a stable resting field and an active field, which was modulated in amplitude but remained otherwise qualitatively constant. The equilibrium point and the pattern of force convergence for the active field did not change through a range of stimulus parameters. When the time-dependent active field and the resting field were summed, we observed a resulting smooth transition of the limb's cquilibrium point to a ncw location and back again. This movement has been called the virtual trajectory (Hogan, 1984, 1985).

The static measurements we used thus allowed us to obtain

\section{Table 3. Comparison of free limb trajectories and virtual trajectories}

Force field and kinematics ( $N=>60$ trials, 12 electrode sites)

$\begin{array}{lll}\text { Different } & 3 & 25 \% \\ \text { Similar } & 9 & 75 \%\end{array}$

Initial kinematics and return kinematics $(N=>255$ trials, 51 sites)

$\begin{array}{lll}\text { Different } & 17 & 34 \% \\ \text { Similar } & 34 & 66 \%\end{array}$

Pause at final position in kinematics $(N=>255$ trials, 51 sites $)$

$\begin{array}{lll}\text { Present } & 12 & 23 \% \\ \text { Absent } & 39 & 77 \%\end{array}$

Data are kinematics in relation to virtual trajectory predictions. Of 51 sites in seven frogs, 12 gave full controls demonstrating electrode stability. At each electrode site at least five instances of the limb kinematics were collected. We also compared relaxation kinematics to the initial motion and examined the kinematics for pauses at the final position. These are summarized for all microstimulation kinematics. "Similar" indicates that paths differ by less than $8 \mathrm{~mm}$ after initial position correction. Stimulation parameters: $300 \mathrm{msec}$ train, $40 \mathrm{~Hz}, 1-7 \mu \mathrm{A}$ an estimate of the virtual trajectory. According to the equilibrium-point hypothesis, this trajectory should predict free-limb paths. Of course, dynamic effects would be expected to alter the actual pattern of motion. To test this notion, we performed the following experiments.

In seven frogs, we stimulated the spinal gray and compared the virtual trajectory with the free limb's trajectory (Fig. 10). In order to measure the free limb's trajectory, we suspended the limb using a sling at the ankle to support it against gravity. This placed the limb in a horizontal plane at a level with the head of the femur. Our examination of the virtual trajectory was confined to this horizontal plane. This arrangement was thus consistent with the focus of our force-field analysis on the horizontal $F x, F y$ components of force. The free limb's trajectory was recorded using a video camera. We set up the camera so as to minimize parallax distortions. The absolute errors in position were estimated to be less than $4 \mathrm{~mm}$; resolution was $\sim 0.4$ $\mathrm{mm}$. The ankle suspension introduced an additional collection of small offset forces due to the sling's action as a pendulum. We took into account the effect of these forces by estimating the pendulum's force field and adding it to the total force field of the limb. In general, this operation resulted in a slight offset to the equilibrium point of the limb's measured force field. We first recorded a set of trajectories, then a force field, then a second set of trajectories. Through this procedure, we ascertained the stability of our stimulation during field collection, trajectory rccording, and the transition between them.

In the majority of instances, our results confirmed that the force fields' virtual trajectories were similar to the free limbs' trajectories. The kinematics of responses were divided into three phases: forward phase (from resting equilibrium to peak displacement), pause phase (during which the limb remained at the peak displacement location), and return phase (from peak 


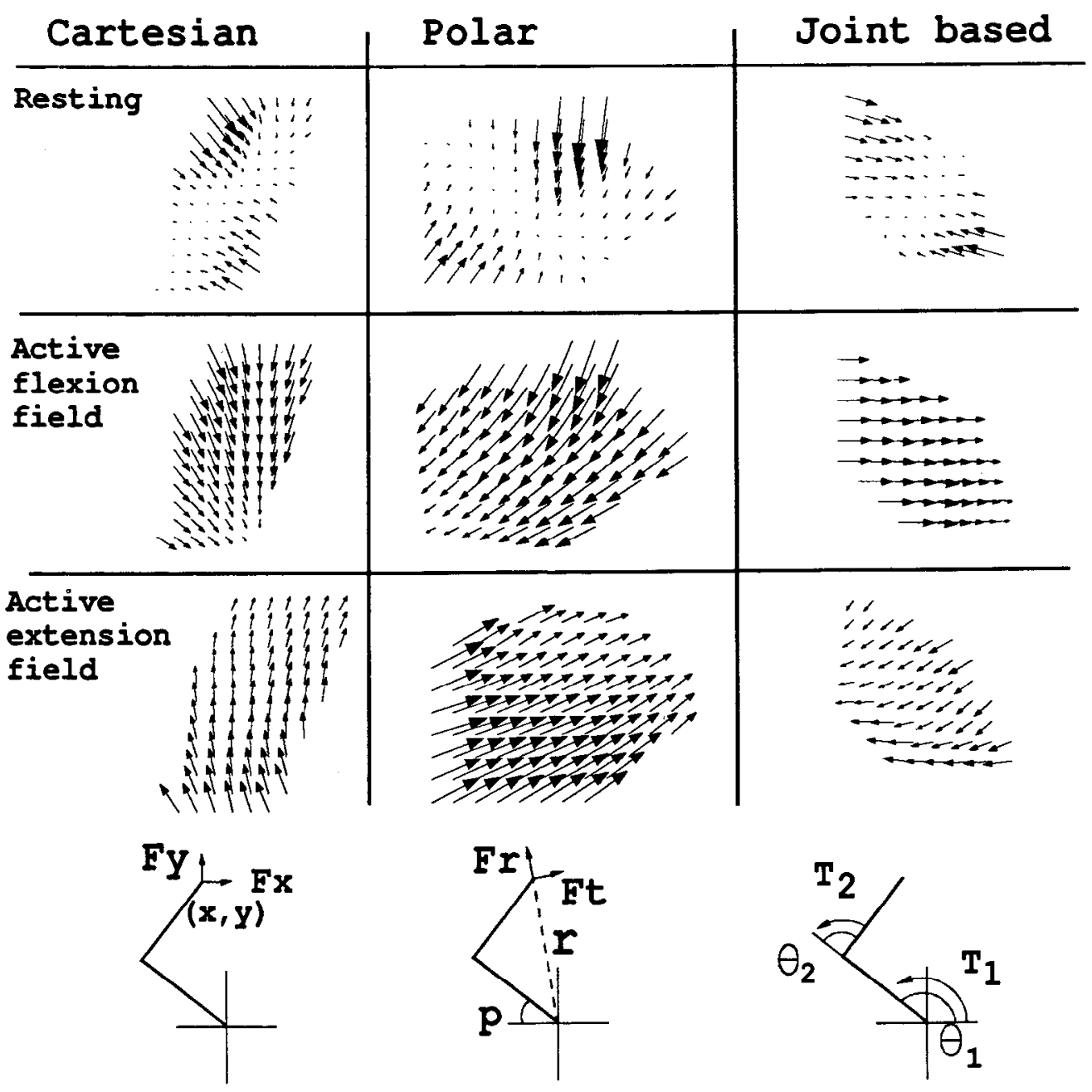

Figure 11. Coordinate systems for expressing force fields. Three fields are shown expressed in three different coordinate systems. The fields are a resting field, an active-flexion field, and an active-extension field. The three fields were all from a single animal. These three fields are expressed in three different coordinate systems. Abscissa and ordinate axes are not displayed. Patterns of the fields should be compared. The abscissa and ordinate axes for the Cartesian coordinate system are in Cartesian space $(x, y)$. The force vectors are end-point force $F x F y$. The abscissa for the polar coordinate system is polar angle $(p)$ and the ordinate is radial distance $(r)$. The vector components are force perpendicular to the polar direction $(F t)$ and polar radial force $(F r)$. The abscissa for the joint coordinate system is hip angle $\left(\theta_{1}\right)$. The ordinate is knee angle $\left(\theta_{2}\right)$. The vector components are hip and knee torque $\left(T_{1}\right.$ and $\left.T_{2}\right)$. The resting field was no simpler in any one of the three coordinate systems in this case. The active flexion shown here had the simplest structure in joint space, suggesting this is a pure hip flexion field (though this was not true of all frogs). The active extension, in contrast, was approximately parallel in polar coordinates. These results typify our data. While in some instances field structures could be simplified in non-Cartesian coordinate systems, this was not truc universally. displacement back to the resting equilibrium). All the observed kinematic responses had a forward and a return phase. However, a pause period (more than $100 \mathrm{msec}$ ) was seen only in $23 \%$ of the cases. In $75 \%$ of our trials in which all controls were satisfied (12 penetrations), we found that despite the small size of the forces induced by microstimulation, the free limb's motion showed a good match to force field predictions. However, in $25 \%$ of these trials, we had repeatable discrepancies between the motion of the limb and the virtual trajectory. We want to stress that our estimates of the equilibrium-point trajectory were based on static measurement of forces with the ankle blocked. It has been demonstrated elsewhere that restraints can occasionally alter spinal-motor programs in the frog (Chepelyugina, 1947; Giszter et al., 1989). In two deafferented frogs that we tested, we never observed such discrepancies. We therefore attribute these occasional differences between actual and virtual trajectory to the particularly strong influences of afference on the cord at some stimulation sites. After cessation of stimulation, the limb returned to the initial posture. In general, this was by the same path, but in $34 \%$ of the cases the kinematics of return differed. Since the kinematics were determined by a relaxing field, this discrepancy is not too surprising. The discrepancies were never radical alterations in path. Statistics for the virtual trajectory matching and the kinematic patterns drawn from experiments involving 51 penetrations are given in Table 3 (these include the trajectories used to compare fields and kinematics).

\subsection{Force description in different coordinate systems}

Our measurements of forces were made in a Cartesian coordinate frame. The choice of this frame was initially dictated by our experimental design. We reviewed our data to make sure that a more suitable body-centered coordinate frame did not exist. In such a frame, the force fields might display a simpler structure related to the control strategies used by the CNS. Accordingly, we examined the force fields we collected in several different coordinate systems: Cartesian, joint space, and polar (Fig. 11).

The Cartesian coordinate system represents the force field as measured by our sensor. The joint-space coordinate system reprcsents the measured forces converted to joint torques and then plotted as a function of joint angle. The torque field might be particularly simple in this angular coordinate system if the spinal cord planned and controlled movement using individual joints. In a few cases, flexion fields became uniform parallel patterns of hip joint torques when expressed in joint space. This result provides an example of single-joint control.

The polar coordinate system we used expresses radial and tangential forces with respect to the hip in the horizontal plane. This coordinate system is a compromise between joint and Cartesian coordinates. Polar coordinates are body centered since they are referenced to the hip, and the coordinate axes change with the configuration of the leg. Unlike joint coordinates, however, the polar coordinates' axes are locally orthogonal across 
A EMG

Figure 12. The variations of EMG activity across a field. $A, \mathrm{~A}$ single EMG record of the semitendinosus muscle collected at a point on the data grid. The associated force field and the tessellation over the data grid are also shown. $B$, Individual records of the semitendinosus at each point in the grid. Note the variations of EMG. Some portion of this variation may be artifactual, but much $(60 \%)$ of the variation in these records was abolished by deafferentation.
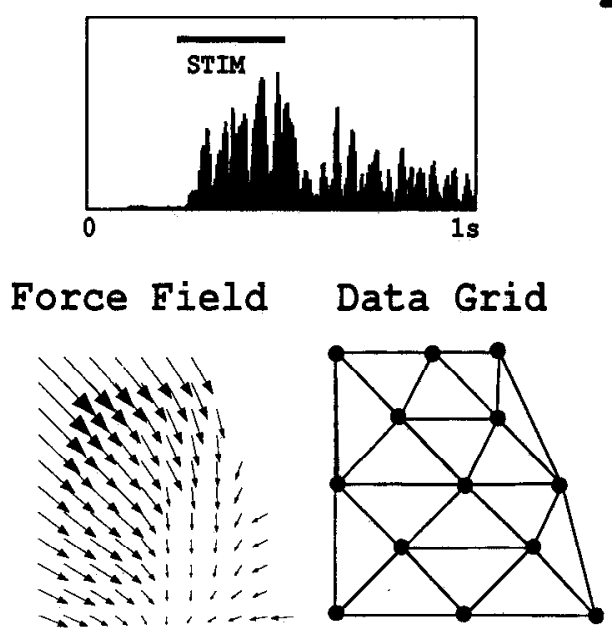

EMG over grid

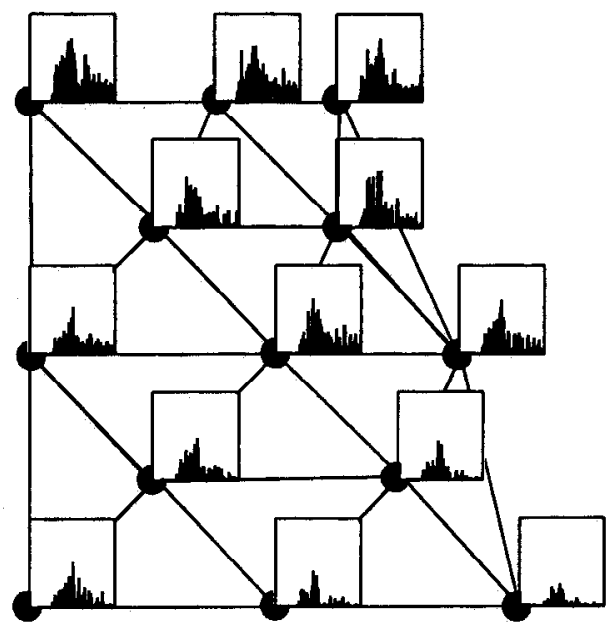

the work space (Flash and Mussa-Ivaldi, 1990). This fact may greatly simplify the spinal cord's control of the limb's end-point. This coordinate system might simplify field descriptions if either hip-centcred planning and control operated, or if stimulation affected only the single-joint muscles at the knee. In some instances, fields were most simply described in polar coordinates. Examples include some passive fields, the active field underlying aversive turning, and some other rostrocaudally oriented fields. In passive fields, the forces around the equilibrium were sometimes fairly isotropic in polar coordinates. The active field underlying aversive turning and other rostrocaudally oriented fields became completely parallel in polar coordinates. However, polar-coordinate descriptions did not simplify the bulk of measured CFFs. After evaluating alternatives, we concluded that no single coordinate system sufficed for all cases, and therefore in general the Cartesian system offered the simplest way to compare the variety of fields. It may well be that different motions of the frog's limb are planned and controlled in different coordinate systems (see, e.g., Giszter et al., 1989; Ostry et al., 1991).

\subsection{EMG activity underlying fields}

In our experiments, we related EMG to the grid location and thus to limb configuration. We also related EMG waveform to the stimulus pulse pattern. This generally failed to produce high correlation for stimulation of regions in the intermediate gray, while electrode placements in the MN pools generated clearly time-locked responses.

Relating the collected EMG data to the grid of configurations over which we recorded our force field data revealed clear variations. Some muscles showed variations of $80 \%$ or more in peak magnitude and in the total area of rectified and integrated response. However, a few muscles also showed very little variation. There were systematic variations in rectified peak magnitude and in the magnitude of the EMG envelope as shown in Figurc 12. In addition, there could be variations in the afferent responses elicited. Many of these happen at stimulus termination. Examples are indicated in Figure 2. We examined EMG peaks and integrated value (envelope) variations in joint-based coordinates, in polar coordinates, and in Cartesian coordinates. We found that the recorded activity generally showed good linear correlation of peak EMG value and integrated (envelope) to
Cartesian position, but a weaker linear correlation to joint or polar coordinate systems. The majority of our analysis has concentrated on Cartesian variation. A linear regression of peak EMG to Cartesian position could account for up to $90 \%$ of the EMG variation. The particular relationships of EMG to position varied from field type to field type, and a given muscle's correlation to $\mathrm{x}$ - or $\mathrm{y}$-coordinate could alter sign in different fields.

The variations are suggestive of the effects of afferent modulation. However, in these experiments muscle activations were close to threshold and thus might involve only a small collection of motor units. The sampling problem for this EMG data was therefore quite severe. We were unable to assess exactly how much of the variation of EMG, at different configurations, was afferent modulation and how much was a result of electrode movement relative to the recorded elements in the muscle. However, since we were able to confirm that more than $60 \%$ of the EMG variation was often abolished by deafferentation, it is likely that a large fraction of the variation reported here was mediated by afferents. The importance of the afferents in defining the pattern of convergence is likely to be small based on data to be presented in section 2.14. The role of afferent feedback in determination of field structure has been addressed more fully in an acute deafferentation study presented in a separate report (Loeb et al., in press).

Attempts to relate collections of muscles seen in the EMG pattern to field type revealed little. The patterns of activity that underlay a field of a particular type within a frog generally showed strong similarity among themselves. Similarly, in a given frog, different fields did have qualitatively different EMG patterns. However, this result did not hold conclusively between frogs. As mentioned, it is likely that a relatively small population of fibers are activated by microstimulation, and our recording electrodes might sometimes have failed to capture the whole pattern of activated fibers when we were operating close to threshold. This source of variation was likely to be accentuated between frogs. Alternatively, it is also possible that different muscle activation patterns might have equivalent mechanical effects. At present, we cannot address these issues with our data.

\subsection{Physiological bases for CFFs}

A priori, there are a number of structures in the spinal cord in which activation might give rise to CFFs. For instance, CFFs 

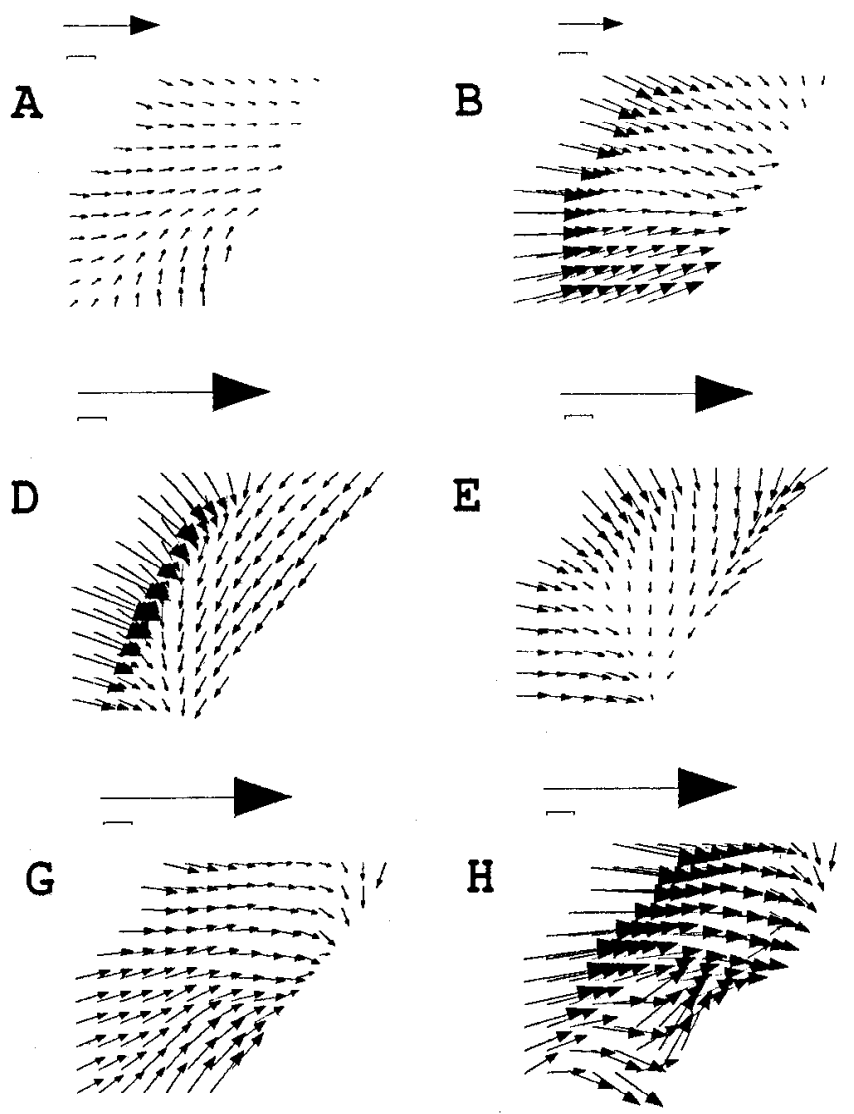

E

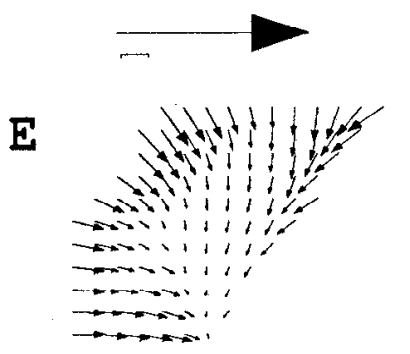

B

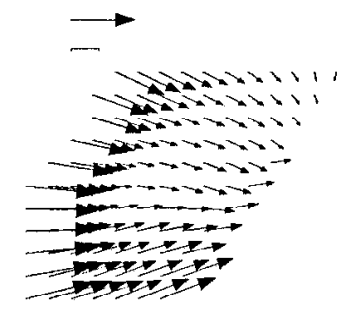

$\mathbf{F}$

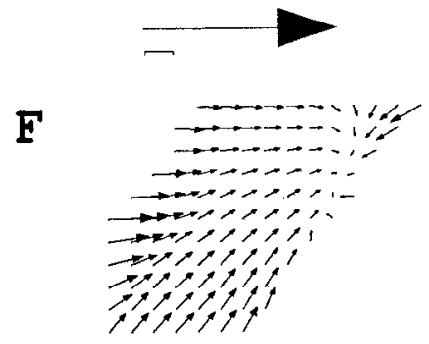

C
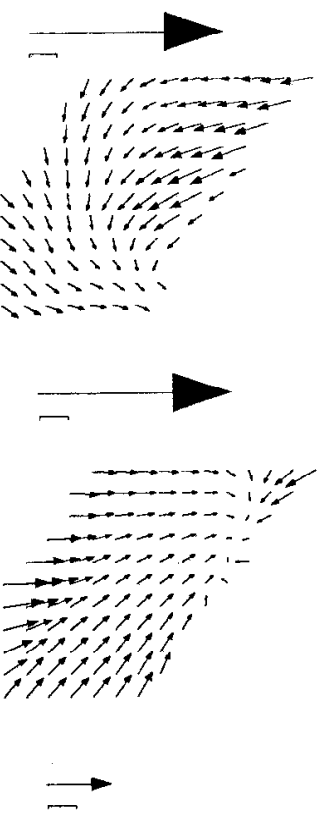

111819181

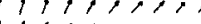

? i

i?

1119

1191?:30

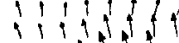

11919lel

inising
Figure 13. Sample active force fields resulting from microstimulation of a frog that had been chronically deafferented over 3 weeks. This figure shows that active convergence of the majority of the force fields elicited in a chronically deafferented frog was preserved. A few fields ( $H$ and $I$ ) wcre lcss well organized following deafferentation. Calibration: $1 \mathrm{~cm}$ and $1 \mathrm{~N}$ in each pane (see Table 4).

might derive from any one of the following three sources: (1) the stimulation of the intraspinal component of sensory fibers; (2) the current spreading to the MNs and to the afferent fibers ending upon them, either randomly or in accordance with their topographic organization; or (3) the activation of long and short propriospinal interneurons. We conducted a set of control expcriments to discover the type of mechanisms necessary for expression of CFFs.

\subsection{Field convergence in chronically transected and deafferented frogs}

We kept chronically transected frogs alive up to 6 weeks before performing microstimulation. Under these circumstances, we believe all descending tracts and synaptic arbors have degenerated based on rates of degeneration of afferent processes (see below). In chronically transected animals, fields were essentially unchanged compared to fields recorded in acutely transected animals.

Because microstimulation evokes patterned activity that might normally be activated by reflex pathways, we examined whether CFFs were critically dependent on an intact afferent system. We discovered that patterned reflex activation is not strictly necessary for the expression of CFFs.

We microstimulated chronically deafferented spinal frogs and found that CFFs with equilibria persisted. To obtain complete degeneration of afferent fibers, we allowed a full 3 weeks after the bilateral section of roots $10,9,8$, and 7 (Joseph and Whitlock, 1968; Glusman et al., 1976; Jhaveri and Frank, 1983). The chronically deafferented frogs were able to move about relatively normally. They could turn accurately from the normal squatting posture, and wiped the back and forelimb successfully both prior to transection of the spinal cord at the base of the brainstem and subsequently (as previously reported in Hering, 1896; Chepelyugina, 1947).

Microstimulation performed in these chronically deafferentfields and their convergent tendency were preserved though some disorganization was seen in a few fields (Fig. 13). In general, field statistics were similar (Tables 2,4). It is known that $\mathrm{MN}$ dendritic sprouting may occur on the time scale of these experiments. However, in another report, we demonstrate similar results and extend them to show identity of force field patterns at a single spinal cord site both before and after acute deafferentation (Loeb et al., in press). Thus, the pattern of afferent impulses and the presence and topography of afferent terminals are not necessary for the generation of CFFs.

\subsection{Muscle organization and recruitment in fields}

2.15.1 Random recruitment of muscle activation patterns. MN dendrites extend long distances throughout the frog's spinal cord (Simpson, 1976). It is conceivable that our convergent fields

\section{Table 4. Deafferented field types}

$\begin{array}{lrr}\text { Active field classification } & & \\ \quad \text { Convergent } & 39 & 93 \% \\ \text { Parallel } & 2 & 5 \% \\ \quad \text { Divergent } & 1 & 2 \% \\ \text { Equilibrium point location } & 8 & 19 \% \\ \quad \text { In test area } & 36 & 81 \% \\ \text { Out of test area } & \end{array}$

Data are locations of equilibria and field types in 42 fields from five chronically deafferented frogs. ed, acutely spinalized frogs showed that both the strength of the 


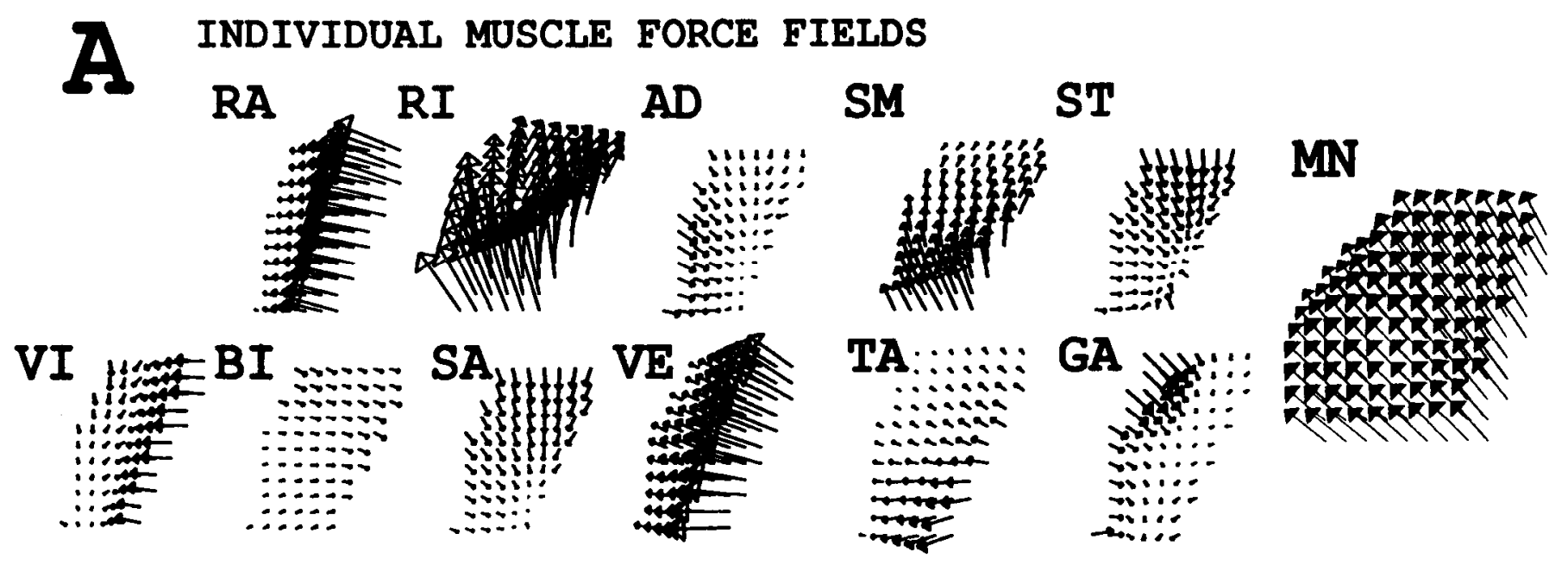

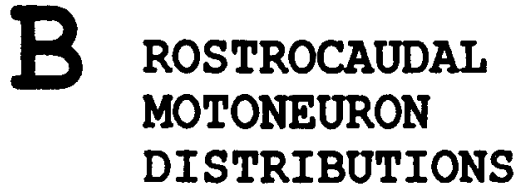
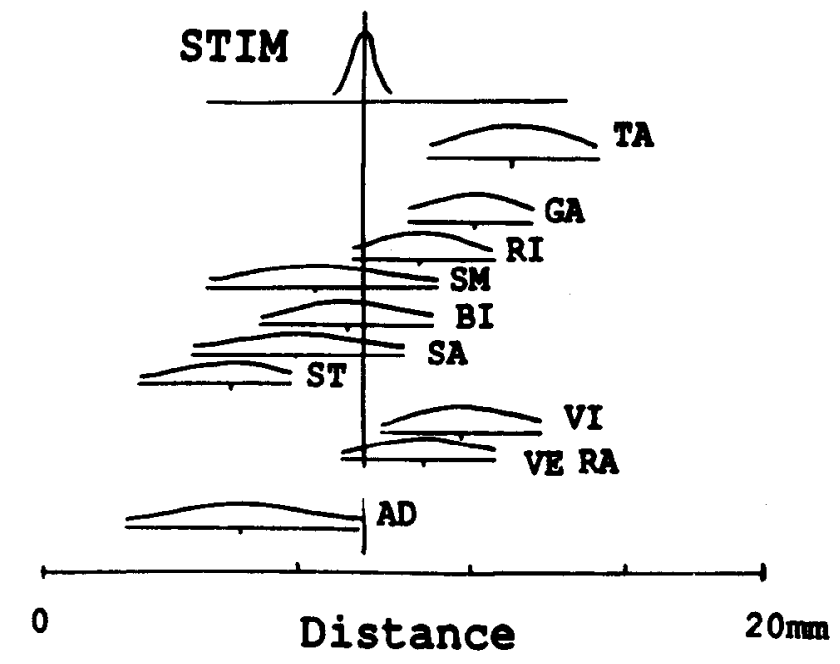

\section{PREDICTED ROSTROCAUDAL FIELD VARIATIONS FROM MOTONEURON DISTRIBUTIONS}

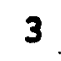

3

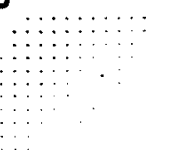

7

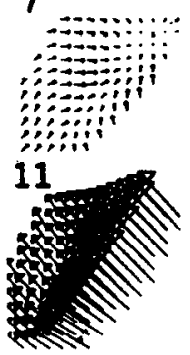

15

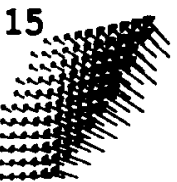

4

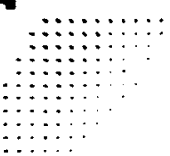

8
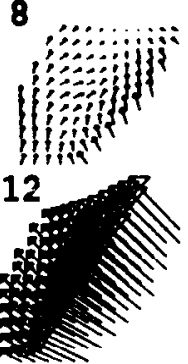

16

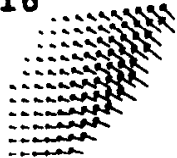

5

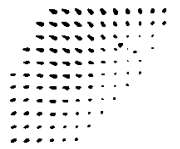

6
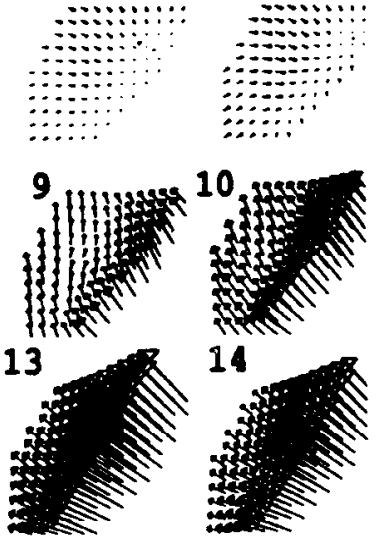

17

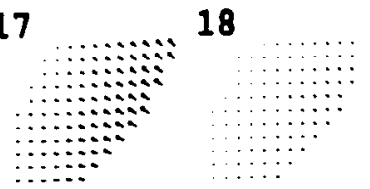

Figure 14. A sample simulation based on muscle fields. $A$, Eleven muscle fields obtained by tetanic stimulation. Stimulation was performed through the pair of electrodes we used for recording EMG. These "muscle" fields formed the basis of our simulations. Muscle labels: VI, vastus externus; $S T$, semitendinosus; $S M$, semimembranosus; $A D$, adductor magnus; $R I$, rectus internus; $R A$, rectus anticus; $V E$, vastus externus; $S A$, sartorius; $B I$, biceps; $T A$, tibialis anticus; $G A$, gastrocnemius. $M N$, A field elicited with the electrode placed among the MNs is also shown. $B$, The distributions of MNs. These distributions were used to estimate the parameters for the summation of muscle fields at a point in the lumbar cord (redrawn from data in Cruce, 1974a). The scale is in millimeters along the rostrocaudal axis ( 0 is rostral). $C$, The force fields predicted by the topographic model. This model used data in $A$ and $B$ (described in Materials and Methods) to predict the fields that would be formed at a particular distance in millimeters along the rostrocaudal axis shown in $B$ (this distance is displayed to the upper left of each field) by direct MN recruitment. This matches MN stimulation quite well in some cord regions (see the example MN in $A$ ).

may have resulted from the random recruitment of MNs either directly or through a random collection of interneurons. Wc therefore tested the hypothesis that CFFs result from the random activation of MNs. Our test consisted in measuring the mechanical responses induced by the electrical stimulation of individual muscles. We stimulated each muscle to maximum tetanus through an implanted pair of EMG electrodes. We collected individual muscle's force fields from $11 \mathrm{leg}$ muscles as described in Materials and Methods. Although there are more than 11 muscles in the leg, those we stimulated represent more than $90 \%$ of the total muscle mass acting on the leg. Next, we simulated random combinations of the 11 measured muscle fields, using the techniques described in the Appendix. Each measured field was modulated by multiplying it with a randomly selected coefficient representing a level of muscle activation. We obtained sets of combined fields by adding together all these modulated fields. This computation simulated a random combination of muscle activations that generated force fields without any afferent control. We found that, over a set of 20,000 simulated combinations, only $8.4 \%$ of fields had an equilibrium point 
within the tested workspace. Thus, random recruitment of MNs could not account for the CFFs that were observed in the majority of our experiments.

In addition, our simulation indicated that the frog's leg muscles constitute a mechanical system biased toward extension: their random combination tended to produce mostly extension forces. In contrast, our stimulation of the premotor layers in the spinal cord resulted in the generation of equilibrium points located predominantly within the work space. Thus, our results suggest that the neural circuits of the spinal cord are organized to compensate for the structural imbalance of the musculoskeletal system.

2.15.2 Topography of $M N$ pools. Data published by Cruce (1974b) demonstrate a topographic organization of the MN pools in the frog. Our results might have derived entirely from such topography. We therefore tested whether CFFs might result from the direct activation of small zones of MNs in the topographically organized MN pools. Extensor MN pools are arrayed caudally to flexor MN pools. However, these distributions overlap, and the various muscles' MN pools are somewhat commingled. We examined to what extent this organization might account for the kind of CFFs we elicited by microstimulation. To do this, we used two methods. First, we used simulations to predict the end-point force fields expected on the basis of the MN distributions. These simulations are detailed fully in the Appendix. In these simple simulations, we assumed no role for feedback. Second, we performed microstimulation experiments with our electrode placed among the MNs' cell bodies.

We assumed that the number of MNs recruited in the vicinity of the electrode is proportional to the spatial density of the MNs at that point in the cord. Thus, to simulate microstimulation at a particular rostrocaudal position in the cord, we combined individual muscle fields after weighting them in proportion to these spatial densities and distance from the electrode location. We examined the resultant end-point force field. In this way, we calculated end-point force fields at a single site in the spinal cord. This calculation was repeated for each point along a rostrocaudal axis in the cord. Although the results matched many of the effects of microstimulation among the MN pools, they could not account for the measured CFFs elicited in intermediate gray (Fig. 14).

In fact, when we placed our microelectrodes directly among the MNs' somas or in the ventral roots, we often obtained force fields with divergent or parallel patterns rather than convergent patterns. In particular, the simulation predicted divergent regions in fields produced by stimulation at caudal locations in the cord. While we found these divergent fields in $\mathrm{MN}$ pool stimulation, we never saw them when we applied upper neuropil stimulation. However, from previous work, we know that stimulation at the $\mathrm{MN}$ soma or in the axon regions is likely to produce different results than stimulation in the intermediate gray (Cruce, 1974a; Gustafsson and Jankowska, 1976). Stimulation among MN somas is likely to differ from stimulation among interneurons in the following features: (1) the balance of fast and slow MNs, (2) the relative levels of transynaptic and direct drive of cells, (3) the neurotransmitter system activated, and (4) the MN recruitment orders.

Our simulation results indicate that the microstimulation of the premotoneuronal circuitry imposes a specific balance of muscle activations leading to a CFF. The simulations also predict that the observed CFFs require precise long-range interactions to balance MN populations. Such interactions cannot be established by current spread or random activation and are likely to be mediated by propriospinal interneurons. The only way we can imagine that the results described here could derive from direct dendritic activation of MNs without mediation of propriospinal interneurons would be if extensive information processing and integration occurred within and between the extensor and flexor MN pools. If such extensive integration occurred, it would be an important motor mechanism in its own right.

2.15.3 Measurement of recruitment patterns. We applied sulforhodamine, a vital dye, to the spinal cord and simultaneously evoked a CFF. We used this technique to examine patterns of activity elicited in the deafferented cord as a result of microstimulation. The sites stimulated were those from which CFFs were elicited. Sulforhodamine is taken up by pinocytosis during reabsorption of synaptic vesicles (Lichtman et al., 1985; Keifer and Houk, 1992). To get sulforhodamine into active cells, we bathed the spinal cord in $0.5 \%$ sulforhodamine for $2 \mathrm{hr}$ while stimulating a single site with a train of pulses every $20 \mathrm{sec}$. The cord was then washed in Ringer for 4 hr and perfused.

It has been remarked by other workers that shortly after intracellular injection of sulforhodamine, the dye is scquestered within the cells and will no longer cross gap junctions. Sulforhodamine taken up from the bathing medium during synaptic activity thus becomes sequestered in the cell either immediately or very shortly after entry. We did not routinely block gap junctions during this study and cannot rule out a limited spread by this means in most of these experiments. However, we do not believe that transmission over gap junctions can acount for the extent of spread of sulforhodamine (over more than $1 \mathrm{~cm}$ ). Furthermore, a control animal bathed in a level of heptanol (10 mM) that was sufficient to block gap junctions showed normal force fields. This animal did have a higher threshold for activity, but sulforhodamine showed the same distributions of activity as animals in our regular experiments.

We obtained clear, broad activation patterns among the MNs in our regular microstimulation regime. In contrast, we observed no sulforhodamine fluorescence in the lumbar cord of controls that were not stimulated. We showed that sulforhodamine uptake depended on the duration of stimulation in the following way. We stimulated one animal for only half the period called for in our standard procedure. In this animal, we observed a more modest fluorescence of a distribution similar to that seen in our regular experiment. Thus, we believe that the sulforhodamine uptake captured the actual breadth of activity in the MN pools following microstimulation. Activity covered up to a centimeter of the lumbar cord. This spread was two orders of magnitude larger than the spread that would be expected from a direct activation by the current from an electrode. In our regular experimental condition, larger $\mathrm{MN}$ dendrites were visible, and in the caudal lumbar cord, some of these dendrites crossed the midline to mingle with dendrites in the contralateral pools. We found sulforhodamine in MNs activated in the brachial cord in many of the spinal frogs. Many also showed a strong forelimb-clasping reflex in our apparatus, and we cannot say how much of the brachial activation was attributable to this reflex. This brachial activation pattern was also visible in control animals.

With respect to the level of fluorescence, there was a range of intensities among the MNs. Adjacent MNs could show very different dyc uptakc. Somc cells positioned among densely labeled pools showed no fluorescence. The activity therefore 


\section{A before loading active peAK field}

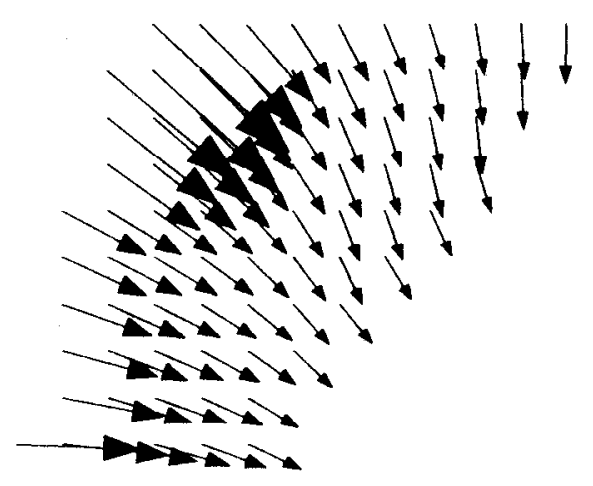

\section{AFTER LOADING ACTIVE PEAK FIELD}

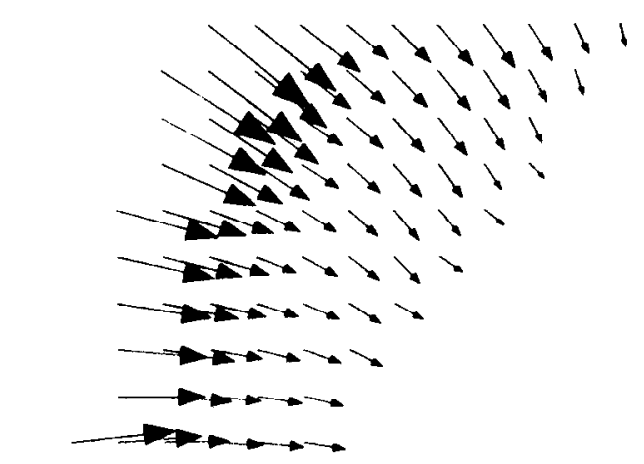

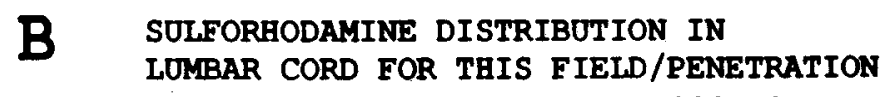

DEPTH about 1200 microns

\section{$400 \mathrm{~ms} 60 \mathrm{~Hz} 0.6 \mathrm{~ms}$ pulses of $2 \mathrm{microAmps}$ DEPTH 700 microns}
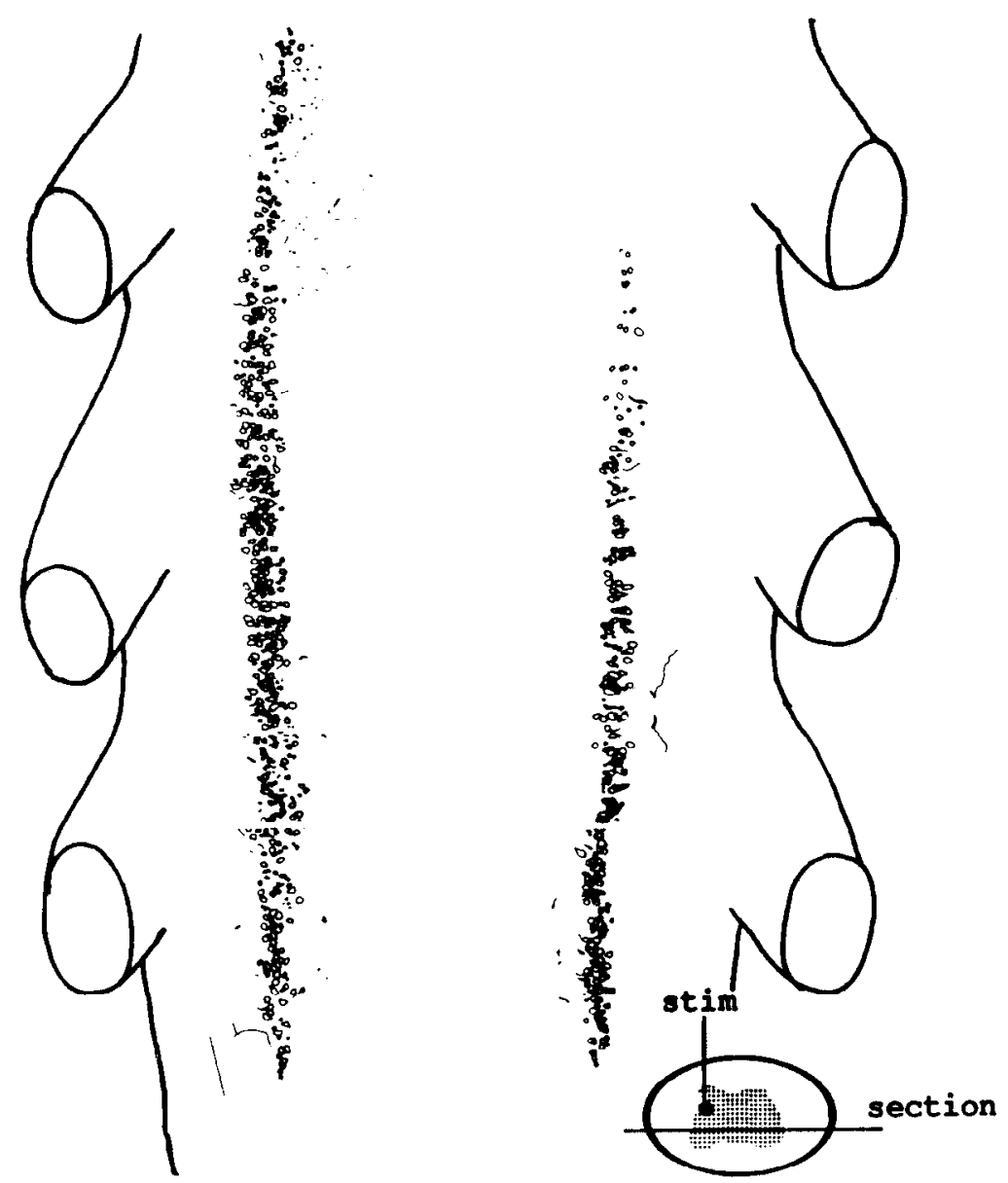

Figure 15. Rhodamine marking of activated MN distributions underlying a field. $A$, The fields recorded before and after the period of sulforhodamine exposure and stimulation. Sulforhodamine was used to mark active cells. The fields were collected to demonstrate electrode stability and characterize the spinal cord site. The field represents a flexion. $B$. Dye-marked, active cells that were visible in the spinal cord in a single horizontal section at around $1200 \mu \mathrm{m}$ depth. (Their size and location indicate that these are MNs.) Note that cells were active on both sides of the spinal cord and over a very broad area covering about $10 \mathrm{~mm}$ rostrocaudally.

seemed to be distributed selectively by interneuronal mechanisms across a breadth of the lumbar cord. Interneurons were also activated in patches, although we have not yet examined these in any great detail. Figure 15 shows a summary of the distribution of $\mathrm{MN}$ activity in a section through the MN region. The associated force field is also displayed.

\section{$2.16 C F F$ and natural behaviors}

We examined behaviors elicited by cutaneous stimulation. Our goal was to determine whether similar convergent and patterned force fields underlie not only the response to focal microstimulation but also natural behaviors. It has been shown that cutaneous and proprioceptive stimulations in the spinal frog produce a range of behaviors: flexion withdrawal, hindlimb-hindlimb wiping, forelimb wiping, back wiping, turning, postural adjustments, and ambulatory motions (Gray and Lissman, 1940, 1946).

By electrically stimulating the appropriate skin areas, we were able to evoke repeatable flexion withdrawals as well as hindlimb wipes and aversive turns. We adjusted cutaneous stimulation so as to avoid habituation or sensitization and to provide a fixed latency response. With our standard method, we then measured the force field: the frog's leg was placed in a number of different work space locations, and we recorded the force vectors induced at each location by a cutaneous stimulation (Fig. 16). During flexion withdrawal, the cutaneous stimulation resulted in force fields that were similar to those obtained by microstimulation (Fig. 17a). We repeated the same procedure for wiping (Fig. $17 \mathrm{~b}$ ) and aversive turning (Fig. 17c). The force fields and equilibrium trajectories from these stimulations showed qualitatively similar patterns to those from the microstimulation of some areas of spinal cord. The equilibrium trajectories also showed a good match to the free-limb kinematics presented in our published data (Giszter et al., 1989).

\subsection{Differences and similarities of natural and microstimulated fields}

It can be seen from a comparison of the records shown in Figure 17, $a$ and $b$, that wiping fields and aversive-turning fields in- 


\section{A: Interlimb Wiping}

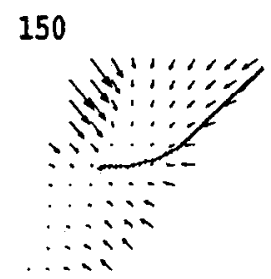

200

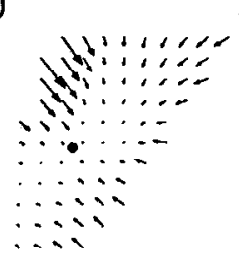

250
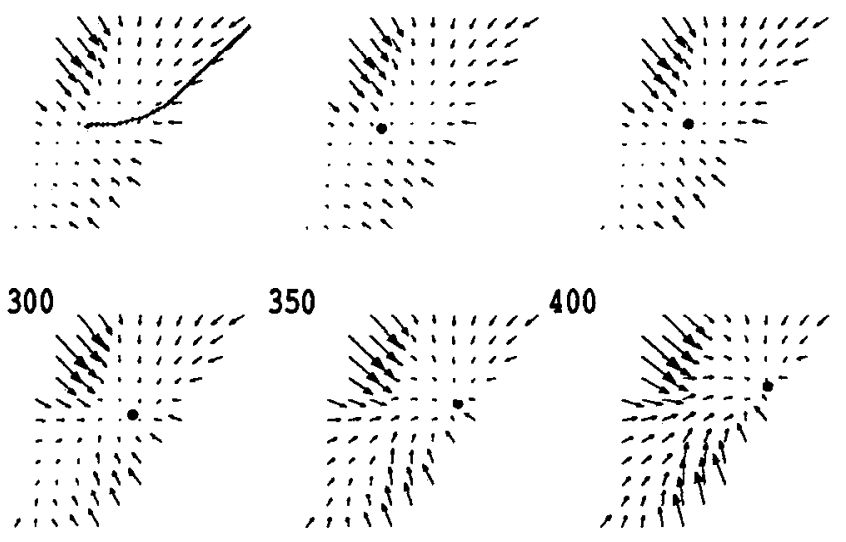

350

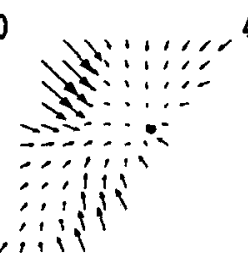

400
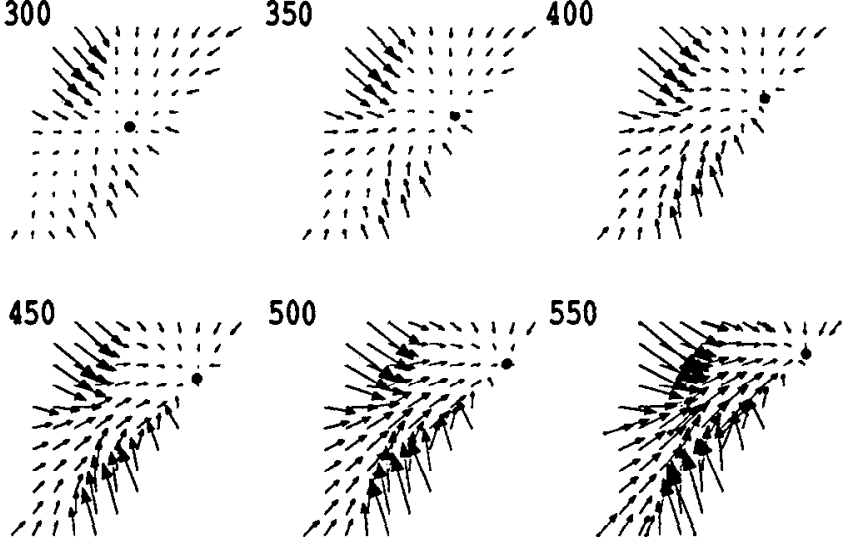

2N
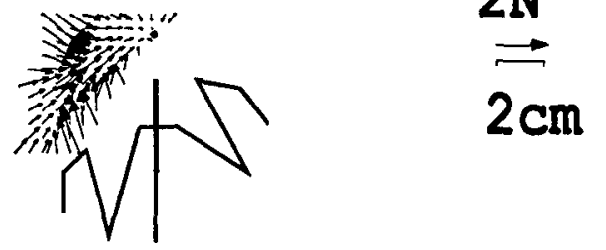
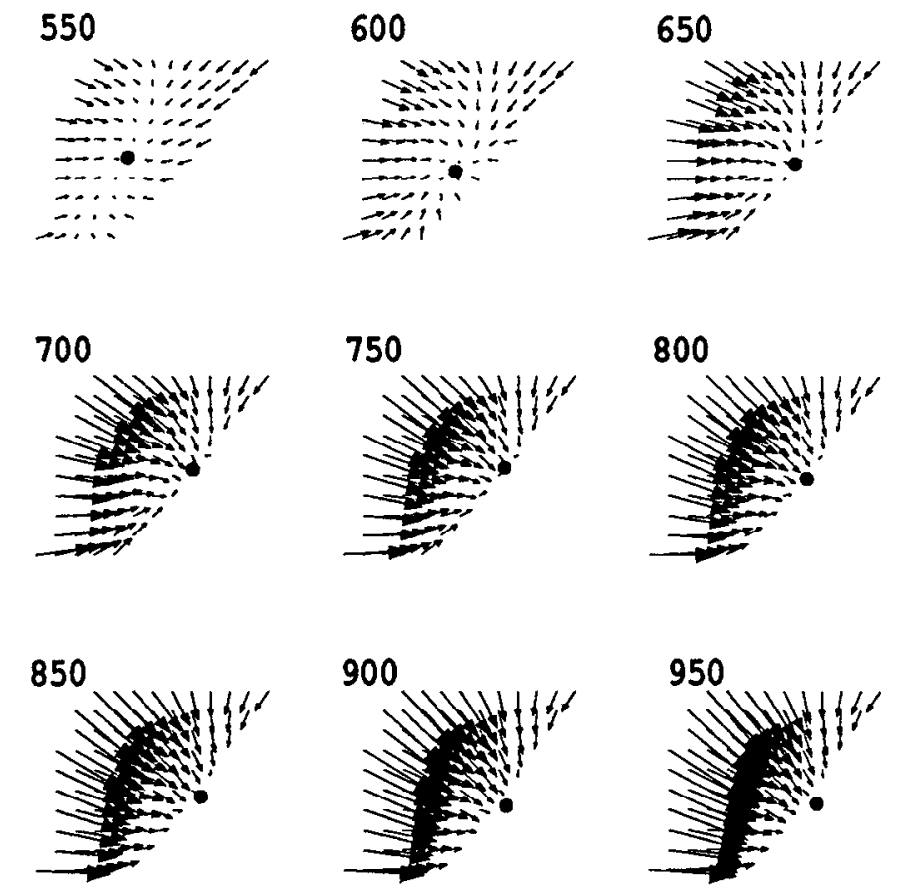

B: Flexion Withdrawal
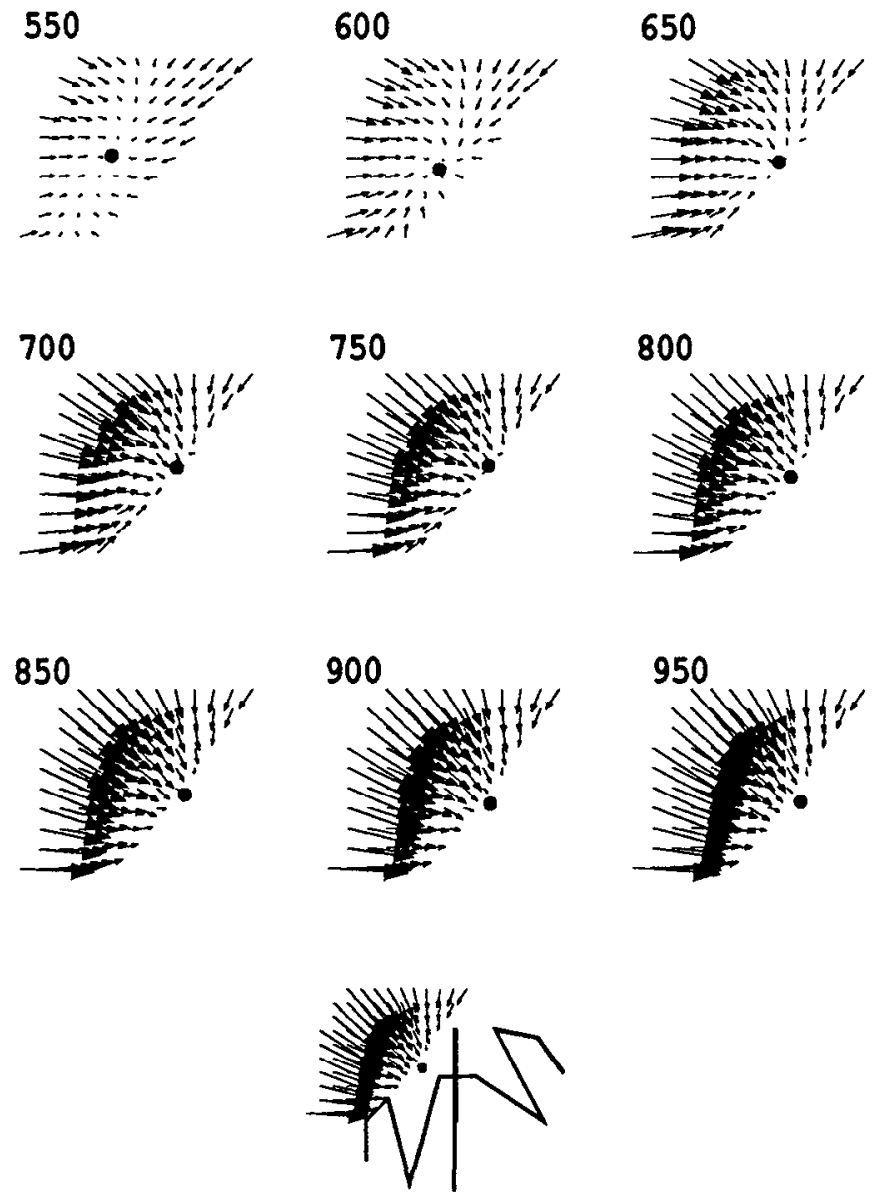

Figure 16. Force fields elicited by cutaneous stimulations. $A$, A force field elicited by electrical stimulation of the heel of the contralateral hindlimb. The time, shown to the upper left of each field, in milliseconds is the time following stimulation onset. The field shows the development of forces over time. The entire virtual trajectory is drawn overlaid on the resting field at $150 \mathrm{msec}$. The path and final equilibrium position of the force field matched results for wiping kinematics. $B$, A flexion force field elicited by electrical stimulation of the plantar foot surface. The time in milliseconds following stimulation onset is shown to the upper left of each field. Below each figure is seen the force pattern in relation to a schematic of the frog's body.

duced by stimulating the skin are similar to some CFFs induced through microstimulation. It should be emphasized that this similarity is particularly evident when a comparison is made between the active field elicited by microstimulation and the active field elicited through cutaneous stimulation. Although there were large differences in the amplitudes of the forces produced, we quantified the similarity between the fields underlying natural behavior and those induced by microstimulation; this operation showed that these two types of fields belong in the same cluster. Active fields underlying natural spinal movements fell in to a number of categories. The majority were convergent. This finding is consistent with the role these fields play in postural adjustment and limb positioning. There was an exception to this naturally elicited field convergence, namely, the active field underlying aversive turns elicited by forelimb stimulation. This field was not convergent, but rather consisted of a curved pattern of forces when expressed in Cartesian coordinates. (This field pattern was completely parallel when expressed in polar coordinates.) This exception is consistent with the field's behavioral role. Aversive turns are propulsive and may not have a goal posture.
The monophasic nature of the force field responses in natural stimulation deserves comment. The leg was immobilized for force field measurement. The afferented animal clearly must have had available the information that the limb did not reach the desired target location (e.g., in the wiping behavior). It has been observed previously in kinematic studies that under thesc circumstances the spinally generated wiping behavior will terminate at this first unsuccessful stage (Giszter et al., 1989). The monophasic response in the force measurement experiments is therefore not unexpected.

In summary, a variety of active fields elicited by microstimulation showed a strong resemblance to the active-force patterns that can be elicited in natural behaviors. These active-force patterns include convergent and parallel active fields, but never divergent fields. The total fields are always convergent. However, we cannot yet provide a one-to-one match between the full repertoire of fields induced by microstimulation and a corresponding repertoire of cutaneously induced fields. It should be stressed that there remain classes of fields induced by microstimulation that display patterns of forces not found so far in the results of cutaneous stimulation. 


\section{A: Hindlimb Wipe B: Aversive turn C: Flexion}

\section{1: FIELDS RESULTING FROM CUTANEOUS STIMULATION}

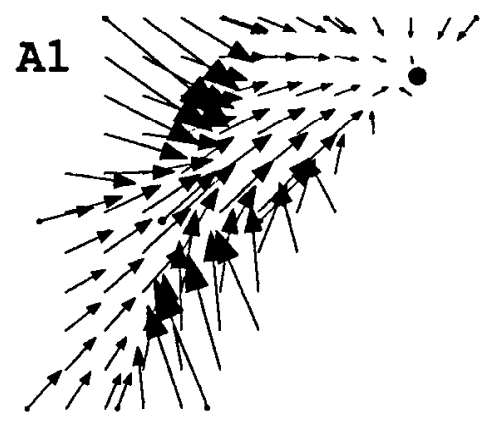

\section{2: FIELDS RESULTING}
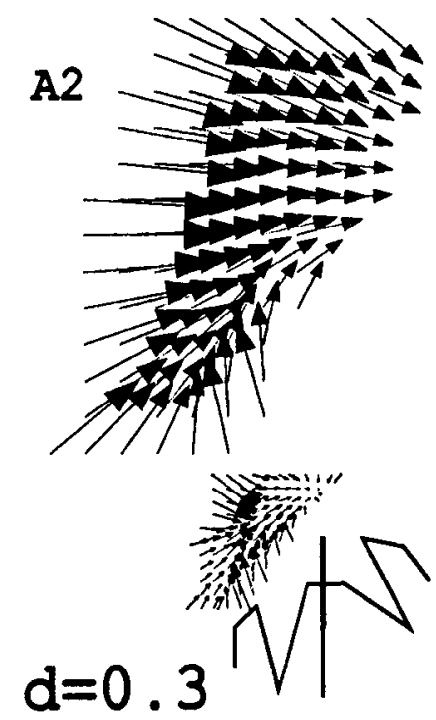

B1

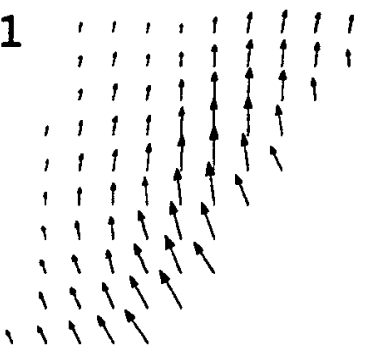

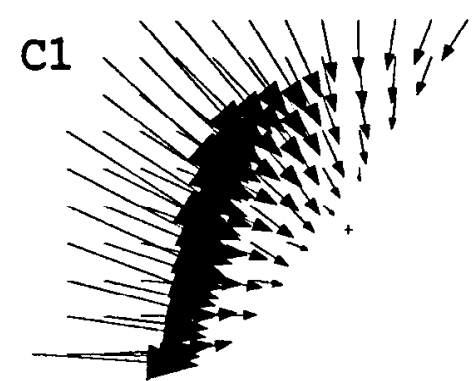

\section{FROM MICROSTIMOLATION OF SPINAL GREY}

\section{B2}
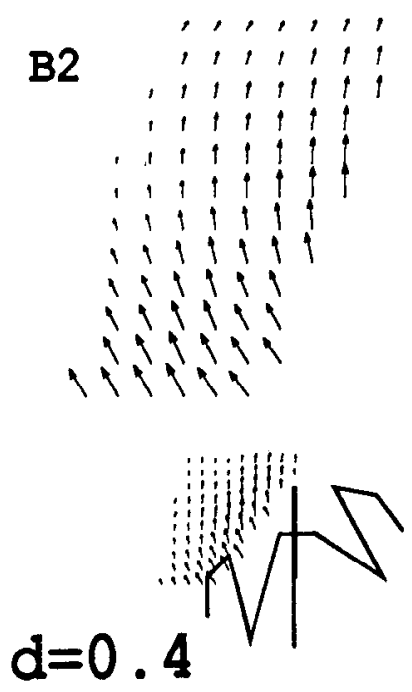
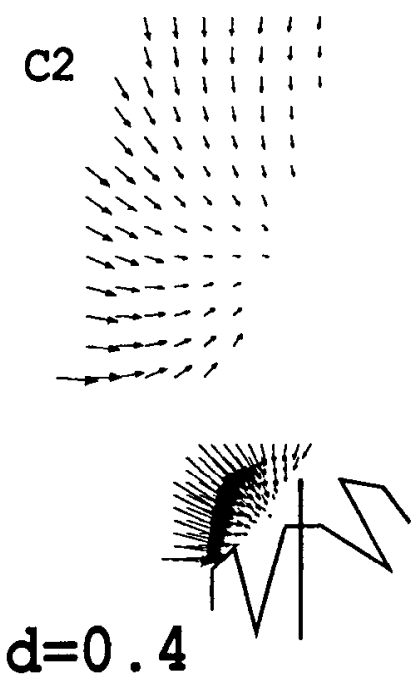

Figure 17. A match of fields was cutaneous stimulation with fields from microstimulation. $a$, Hindlimb-hindlimb wiping field pattern elicited by cutaneous stimulation of contralateral hindlimb (a1) and microstimulation results elicited in spinal gray (a2). Note that the field in (al) covered a slightly larger area than the field in $(a 2)$. Similarity measures $(d)$ of less than 0.5 indicate fields are to be placed in the same class. Similarity was calculated from Equation 4. $a$, Aversive turning field pattern elicited by cutaneous stimulation of ipsilateral forelimb (bl) and microstimulation results elicited in spinal gray $(b 2)$; similarity measure 0.4 . $c$, Flexion withdrawal field pattern elicited by cutaneous stimulation of foot $(c 1)$ and microstimulation of spinal gray $(c 2)$; similarity measure 0.4 . These two different instances of each of the three field types are, by our similarity criterion, identical.

\section{Discussion}

The main results of our investigations are threefold. First, we have shown that microstimulation of different areas of the intermediate gray regions of the frog's spinal cord produces a small set of different types of well-organized force fields. Second, we have shown that similar fields underlie the generation of natural behaviors like wiping patterns and flexion withdrawal. These two types of fields have a number of interesting properties in common, the principal one being convergence of the total field toward a single equilibrium location. Finally, we have shown that divergent fields result from direct MN activation in the caudal lumbar cord but that such fields are never seen in stimulation of intermediate gray.

\subsection{Microstimulation in the spinal cord}

Microstimulation has been used with good results in the mammalian spinal cord to examine connectivity and circuit behavior (Gustafsson and Jankowska, 1976). Nonetheless, it must be ac- knowledged that activation by microstimulation lacks speciticity. For this reason, it is perhaps surprising that our force field results showed such consistency and repeatability without our using special additional constraints on location. The previous body of work suggests that at the currents we used in the intermediate gray, by far the greatest effects of stimulation are likely to be transynaptic (Gustafsson and Jankowska, 1976). This previous work also suggests that it is unlikely that the stimulation could have directly activated fibers in the white matter from the spinal cord locations at which we collected fields in this study.

\subsection{Active field characteristics}

We routinely separated our data into resting fields and the stimulus-generated active fields. We observed that after the removal of resting forces, the wide variety of field structures and equilibrium positions was considerably reduced. This separation into active and resting fields is partly justified by the division of the frog musculature into two fiber classes operating in very 
different ways. The slow-fiber system is tonic, graded, and characterized by polyneuronal innervation. It operates on a long time scale. Conceivably, the slow-fiber system provides the physiological underpinning for the resting field (Simpson, 1976; Morgan and Proske, 1984). Our microstimulation of the cord activated the twitch system, as shown by the EMG patterns (slow-fiber system is nonspiking) and the time course of force development. We hypothesize that the twitch system activity was overlaid on the resting field. By focusing on the active field, we simplified the description and interpretation of our data.

The active fields were found to be nearly structurally invariant. By the term "structurally invariant," we intend to indicate that the orientation and balance of forces (and hence the positions of any equilibria) remained constant both through time and for a range of variations in the stimulus parameters. However, the active fields were scaled in amplitude. The fields elicited by different stimulus strengths and durations at a single spinal locus were amplitude-scaled versions of one another. Presumably, all fields of a given type were scaled versions of the same overall spatial pattern of control. The variety of active fields exhibited in sampling the spinal cord was small. This classification into types was confirmed by a cluster analysis. The small number of distinct field types and the constant organization of forces suggest that these fields and the underlying synergies represent fundamental elements of spinal-motor organization.

\subsection{Active field as a movement primitive}

The synergies described here are coordinative groupings that generate stable force fields in the limb. We believe that these active CFFs may be viewed as fundamental units of motor behavior, or "movement primitives" (Giszter et al., 1992a,b). By the term "movement primitives," we mean indivisible elements of motor behavior that generate a regulated and stable mechanical response. This organization of motor behavior may have several advantages. Movement primitives might form a basis for the effective combination of sensory and descending controls. Presumably, an organization of CFFs or movement primitives supported by specific muscle synergies may allow the CNS to use simpler feedback schemes. Thus, the problem of incorporating novel information from descending pathways or from different types of sensory afferents for motor learning would be simplified. This is the problem of "sensor fusion" that has sometimes presented difficulties in robotics. Finally, by modulating a fixed synergy with well-specified mechanical effects, the CNS might combine several different sets of inputs without additional computation, so as to produce a coherently modulated limb response. This framework is clearly an extension of the classical concepts of reflex alliance and coordination due to Sherrington (see Sherrington, 1961) and superposition due to von Holst (see von Holst, 1973).

\subsection{What are the potential uses of CFFs or movement primitives?}

Work described here and elsewhere (Bizzi et al., 1991; Giszter et al., 1991a,b, 1992a,b) suggests that there may be a limited number of force field types available in the spinal cord of the frog. Thus, to obtain a repertoire of adjusted movements, the CNS must combine or enhance these primitive elements in various ways. We envisage several mechanisms that might enlarge the repertoire of outputs based on force fields: (1) indi- vidual force fields might be modulated directly by descending pathways or indirectly by modifying afferent effects (altering control of the synergy); (2) force fields might be altered by overlaying additional novel muscle activity (altering the composition of the synergy); (3) several force field types might be combined together (combining synergies).

In other publications, we have presented preliminary tests of the third alternative (Bizzi et al., 1991; Giszter et al., 1992a). We found that multiple stimulations in two different zones produced well-organized fields in two dimensions. The stimulation of two sites simultaneously resulted in a field proportional to the vector sum of the two fields elicited singly. This mechanism is consistent with the vector summation of muscle fields that was used in the simulations presented here. Thus, a "blending" of the two fields elicited in different zones can occur. This finding leads us to the speculation that a vector-field combination might form the basis of the kinematic blending that has been observed in the turtle scratch (Mortin et al., 1985; Robertson et al., 1985; Stein et al., 1986) and the frog's wiping (Berkinblit et al., 1989). In fact, recent simulation work (Mussa-Ivaldi et al., 1991a,b) has shown that the combination of a small number of fields with different equilibrium points can be used as a mechanism to generate a wide variety of different force fields (and thus behaviors).

\subsection{Muscle patterns and control underlying CFFs}

At present, it is difficult to assess the strength of afferent influences on the microstimulation-based force fields recorded here. The large modulation of the EMG that we observed suggests that the influence of afference may be considerable. However, we cannot dismiss the hypothesis that these variations are often due to inadequate sampling and electrode movement in different configurations. Similarly, assessment of the qualitative muscle activation patterns associated with fields was also difficult. Although we have identified a few types of force fields, we cannot assess whether these few types are due to a few fixed synergies. The possibility remains that these fixed force fields are organized by several different motor-equivalent (Bernstein, 1967) muscle patterns and feedback strategies.

\subsection{Circuilry supporting $C F F$}

By a process of elimination, we have reduced the sets of circuits that we believe are strictly necessary to support the CFFs: the necessary circuits are intrinsic spinal interneurons and MNs. We have shown that afference is not essential to the expression of a CFF, by examining frogs with chronic bilateral deafferentations. However, it is clear that the sensory afferents must play a role in tuning CFFs.

By an examination of frogs with chronic transections, we know that the presence of descending terminal arbors is not strictly necessary to the expression of CFFs. Our results still leave a formidable array of spinal mechanisms as candidates for the generation of CFFs. In all likelihood, the MNs contributing to the CFFs are activated by a circuitry that includes spinal cord interneurons, the propriospinal system, the interneuronal targets of the descending systems, and perhaps interactions among the MNs' extensive dendrites. At this time, we do not know which of these elements is the most significant for the production of the CFFs by microstimulation.

We explored some of the most straightforward hypotheses of CFF production. Using a number of simulations, we examined 
the possiblc cffcets of activation of the rcmaining circuit clcments. These simulations showed that neither the $\operatorname{limb}$ and muscle geometry nor the simple topographic organization of MNs accounts for microstimulation-based CFFs. However, we do not know to what extent the organization of CFFs may depend on dendritic processing in the MNs, inter-MN electrical and chemical connectivity, and intrinsic MN membrane properties. Despite this, we expect a large role for interneurons in distributing the activity underlying CFFs. We base this expectation on the following results: (1) the broad regions from which qualitatively similar fields can be elicited, (2) the breadth of MN activation demonstrated by sulforhodamine, and (3) the demonstrable balance of muscle activations. The results reported here are based on penetrations with the electrode in the center of the gray matter. Different types of circuits and mechanisms may underlie some of the range of field variations we have seen. We have seen variations of field types when we moved the electrode mediolaterally in the cord (Bizzi et al., 1991). Different types of neuropil may have been stimulated by the electrode in these mediolateral locations.

The activated MNs underlying the CFF synergies were shown to be distributed through a broad region of spinal cord. Despite this breadth of activation, we believe that the pattern of activation within the $\mathrm{MN}$ pools is finely balanced. Broad nonspecific activation of the MN pools in simulations does not generate the types of fields we have observed. Additionally, the sulforhodamine results demonstrate that of two adjacent MNs in a region of cord, one could be highly active and the other silent.

\subsection{Relationship to other spinal cord work}

Our results should be viewed and interpreted in the context of other work on spinal cord mechanisms. It is important to consider the evolutionary position of the frog. In the frog, evolution provides a particular type of "reduced" vertebrate preparation. The organization of control structures found in the spinal cord of the mammal probably represents a compromise between various learned movement optimizations, computational constraints, and the structural constraints imposed by the evolutionary and developmental processes. A primitive ancestral spinal cord formed the substrate on which the more sophisticated control mechanisms of the mammalian organization were eventually layered through evolution (see Cohen, 1988). While this ancestor is not available, the frog's spinal cord represents a reasonable system in which to examine the motor control adaptations and limitations that may have existed historically in the primitive and anamniote tetrapod cord.

The frog spinal cord differs from the mammalian cord in several respects that may affect both local and descending control strategies. The finely differentiated laminar structure of the mammalian cord is missing. In contrast, only two areas of cutaneous and proprioceptive afferent arbors can be easily distinguished in the areas we usually stimulate (Cruce, 1974a,b; Jhaveri and Frank, 1983). The gamma fusimotor system is completely absent in the frog. There is no corticospinal tract in the frog. The frog MN arbors are more distributed in the cord than in the mammal (Simpson, 1976). The frog's muscular system contains a system of slow fibers that respond in a graded fashion and may be purely postural. Lastly, the behavioral repertoire of the frog is simpler.

Thus, the frog's spinal cord represents a particular functional organization of circuits and afferent controls that are a subset of the full gamut of controls found in the mammalian motor system (sec Houk, 1972). Our work may help to assess the function of these subsystems, in particular the beta fusimotor system. The roles of the beta fusimotor system in posture and movement have not received as much attention as has the gamma system. The role of this beta motor organization in the control of CFFs may provide some important insights.

This work also has clear ties to prior research on spinal mechanisms by Alstermark and Sasaki (1986) and Gustafsson and Jankowska (1976). These investigators used lesions, recording, and stimulation to examine the roles of circuits in behavior and the connectivity and functional organization of subsets of spinal circuits. Gustafsson and Jankowska used microstimulation to examine local circuit structure and local connectivity. Our results here describe the global mechanical force responses elicited by similar microstimulations. Our approach provides both a direct measurement of these responses, and a framework for the analysis of them. Our work may cventually provide a bridgc between both the behavioral and the circuit descriptions by describing the overall control and biomechanics implemented by collections of circuits.

\subsection{CFFs and natural behaviors}

We have shown that structured CFFs almost identical to those induced by microstimulation underlie cutaneously elicited behaviors in the spinal frog. A natural question, therefore, is what is the relationship of the circuits or movement primitives activated in cutaneously induced behaviors and the circuits underlying the CFFs generated by microstimulation. We imagine three possible relationships between these microstimulationbased circuits and natural behaviors in the spinal cord. (1) The microstimulation may simply induce a behavior just as cutaneous stimulation would. (2) The microstimulation may activate a subset of the circuits of a complete behavior, but this subset of circuits may be inaccessible to descending pathways and other circuitry. (3) The circuits activated by microstimulation may be shareable subunits of natural behavior (or movement primitives). Shareable subunits might be used in several natural behaviors either in isolation, in sequential chains, or in parallel combination with other such circuits. If the latter were the case, we speculate that the CNS could use such a mechanism to transform the various sensory and intermediate representations found in the higher nervous system structures (e.g., Georgopoulos et al., 1988a,b; Masino and Grobstein, 1989; Grobstein, 1990; Masino and Knudsen, 1990) into muscle activations and movement patterns (see Bizzi et al., 1991; Mussa-Ivaldi and Giszter, 1991; Mussa-Ivaldi et al., 1991a).

Despite the phylogenetic relationships discussed above, we do not know exactly how the propriospinal, supraspinal, and pattern-generating circuits described in other species relate to the microstimulation-based CFFs. Because we have found only a few types of active CFFs, we believe that the premotor regions of the spinal cord contain fairly large areas that are functionally equivalent with respect to the generation of active CFFs. This notion is reflected in our observation that a given type of active CFF can be elicited from loci that are several millimeters apart. Preliminary results described elsewhere indicate that the circuitries responsible for CFFs of a particular type are grouped together in distinct areas (see Biz.zi et al., 1991).

\subsection{Equilibrium-point control strategy}

In principle, the evolution of the isometric force field that we measured can specify a limb trajectory. When we compared 
force-field equilibrium trajectories with actual movements, there was a remarkably good match. Our point of suspension introduced an offset field to the limb forces. When these offsets were taken into account the match was quite good in $60 \%$ of trials and an additional $15 \%$ were reasonable. However, some $25 \%$ of responses showed differences between kinematics and force responses. We currently attribute these discrepancies to switched spinal behavior strategies due to detection of the constraint imposed by the force sensor. There was also a good match between force field structure and natural behavior kinematics. These results show that in the spinal frog, an equilibrium-point strategy underlies most of the movements generated by cutaneous stimulation as well as by microstimulation.

The results reported here are thus clearly related to the equilibrium-point hypotheses. This hypothesis was originally formulated by Feldman $(1979,1986)$, who proposed that the limb's posture derives from the interactions between the spring-like properties of opposing muscles. According to the equilibriumpoint hypothesis, limb movements result from a shift in the equilibrium point (see Bizzi et al., 1992). The studies described here provide a degree of neurophysiological and biomechanical support for this hypothesis. We have shown that the spinal cord contains circuitries whose activation by microstimulation produces balanced and spatially modulated contraction in groups of muscles. These sets of synergistic contractions generate forces that, taken in total, direct the limb toward an equilibrium point in space. A similar strategy of muscle use underlies the force field patterns that support wiping and flexion withdrawal when they are induced by cutaneous stimulation. These two sets of patterns were elicited in very different ways. It is remarkable that the two patterns seem to be based on similar underlying equilibrium-point strategies.

The CFF mechanism described here thus has implications both for the control and the computation of movements. With respect to control, the elastic properties of the muscles provide instantaneous correcting forces when a limb is moved away from the intended equilibrium by some external perturbation. With respect to computation, the same elastic properties may offer the brain an opportunity to deal with the inverse-dyanmics problem. Once the brain has achieved the ability to represent and control equilibrium postures, it can master movements as temporal sequences of such postures. The use of an equilibriumpoint strategy may be a characteristic of spinally generated motor programs within the frog's spinal cord. Furthermore, equilibrium-point control may represent a fundamental strategy of the spinal execution of movement in general.

To conclude, this work suggests that the spinal cord possesses the ability to generate a small number of convergent and patterned force fields, or movement primitives. These elements may support equilibrium-point mechanisms and may generate movement trajectories. Some of these force field types clearly also underlie phases of natural behaviors. We believe that these force field primitives are therefore used in pattern generators and other spinal behaviors, and are recruited by descending control systems to produce adjustable behaviors.

\section{Appendix}

Equation numbering in the Appendix continues from numbers of equations in main text.

A.1 Muscle field analysis. The force fields induced by direct muscle stimulation were described in the following mathematical framework. We indicate a position of the ankle in the work space by $\mathbf{r}-(x, y)$, and a configuration of the hip $\left(q_{1}\right)$ and knee $\left(q_{2}\right)$ by $\mathbf{q}=\left(q_{1}, q_{2}\right)$. The kinematic constraint imposed on the frog's leg by our apparatus implies that in the tested region of the ankle work space, there exists an "inverse kinematic" function

$$
\mathbf{q}=\phi(\mathbf{r})
$$

that forms a one-to-one (and bijective) map from ankle locations into joint configurations. A given joint-torque vector, $\mathbf{T}=\left(T_{1}\right.$, $\left.T_{2}\right)$, corresponds to an ankle force vector, $\mathbf{F}=\left(F_{x}, F_{y}\right)$, which depends upon the ankle as

$$
\mathbf{F}=\mathbf{A}^{T}(\mathbf{r}) \mathbf{T} \text {. }
$$

In the above expression, the matrix $\mathbf{A}(\mathbf{r})$ is the position-dependent Jacobian of the inverse kinematics relation (Eq. 5), that is, $\mathbf{A}(\mathbf{r})=\delta \phi / \delta r$. In practice, the matrix $\mathbf{A}$ was computed at all (nonsingular) points by inverting the Jacobian of the direct kinematic transformation $\mathbf{r}=g(\mathbf{q})$.

The isometric tension, $f_{t}$, developed by the $i$ th muscle is a function of the muscle's length, $l_{i}$, and the muscle's activation. Here, we described each muscle's activation by a single lumped variable, $u_{i}$, whose value may range continuously from 0 (resting) to 1 (maximal activation). Thus, the isometric tension is given by

$$
f_{i}=\mathbf{X}_{i}\left(l_{i}, u_{i}\right)
$$

The length, $l_{i}$, of the muscle is related to the joint configuration q by the function

$$
l_{i}=\rho_{i}(q),
$$

whose Jacobian $\mu_{i}=\delta \rho_{i} / \delta \mathbf{q}$ is the matrix of moment arms. This matrix is used to derive the torque generated by a muscle at all joint configurations as

$$
\mathbf{T}\left(\mathbf{q}, u_{i}\right)=\mu_{i}^{T}(\mathbf{q}) X_{i}\left(\rho_{i}(\mathbf{q}), u_{i}\right) .
$$

By combining Equations 5-8, we were able to describe the net mechanical effect of a muscle activation as a field of ankle forces:

$$
\mathbf{F}_{i}\left(\mathbf{r}, u_{i}\right)=\mathbf{A}^{T}(\mathbf{r}) \mu_{i}^{T}(\phi(\mathbf{r})) X_{i}\left(\rho_{i}\left(\phi_{i}(\mathbf{r})\right), u_{i}\right) .
$$

The above expression is contingent upon the assumption that the inverse-kinematic function $\phi(\mathbf{r})$ is uniquely defined. This was indeed the case in our experimental setup.

The net force field at the ankle generated by a set of $N$ muscles with activation levels $u_{1}, u_{2}, \ldots, u_{N}$ was obtained by summing the individual muscle fields; that is,

$$
F\left(r, u_{1}, u_{2}, \ldots, u_{N}\right)=\sum_{i=1}^{N} F_{i}\left(r, u_{1}\right) .
$$

In this context, the resting field $F_{R}(\mathbf{r})$ is by definition the field obtained when all the activation variables are zero:

$$
F_{R}(\mathbf{r})=F(r, 0,0, \ldots, 0)=\sum_{i=1}^{N} F_{i}(r, 0) .
$$

Hence, if we set

$$
\Delta F_{i}\left(\mathbf{r}, u_{i}\right)=F_{i}\left(\mathbf{r}, u_{i}\right)-F_{i}(\mathbf{r}, 0),
$$

the net field can be written as

$$
F\left(r, u_{1}, u_{2}, \ldots, u_{N}\right)=F_{R}(r)+\sum_{i=1}^{N} \Delta F_{i}\left(r, u_{i}\right)
$$

The above expression has a practical value since it allows one 
to estimate a single muscle's contributions to the net field. In fact, the selective stimulation of muscle $k$ corresponds to setting to zero all the activation coefficients except $u_{k}$ :

$$
F\left(\mathbf{r}, 0, \ldots, u_{k}, \ldots, 0\right)=F_{R}(\mathbf{r})+\Delta F_{k}\left(\mathbf{r}, u_{k}\right) .
$$

We used this expression for estimating the maximal active contribution $\Phi_{i}(\mathbf{r})=\Delta F_{i}(\mathbf{r}, 1)$ of individual muscles to the total field. To this end, we subtracted the resting ficld, $F_{R}(\mathbf{r})$, from the field measured while stimulating muscle $i$ at the maximum level of tetanic tension we could achieve with stimulation through our recording electrodes. In our model, we have assumed that the contribution of each muscle could be expressed as

$$
\Delta F_{i}\left(\mathbf{r}, u_{i}\right)=u_{i} \Phi_{i}(\mathbf{r}), \quad u_{i} \text { in interval }[0,1] .
$$

Random muscle fields were simulated to test whether random dendritic activation of MNs could produce our observations. These were simulated as

$$
F\left(x, c_{1}, c_{2}, \ldots, c_{N}\right)=F_{R}(r)+\sum_{i=1}^{N} c_{i} \Phi_{i}(r),
$$

with a set of pseudorandom coefficients $c_{1}, \ldots, c_{N}$ in the interval $[0,1]$.

Once again, it should be emphasized that this procedure was only feasible because of the particular recording arrangement and limb constraints utilized in these experiments. With this procedure, we obtained a collection of muscle force fields that formed the basis for simulating models of various feedforward activation patterns for MN pools and muscles. In contrast, in the unconstrained redundant limb, the end-point force resulting from a collection of muscles could not be obtained in this simple way, and would depend on the coactivation pattern in a complicatcd nonlincar fashion.

A.2 Motoneuron topography model. We examined the effects of MN topography using a simple model. In order to model the direct recruitment of motoneurons close to the electrode, we made a number of simplifying assumptions.

(1) We assumed the fast and slow MNs of an individual muscle were similarly distributed in the pool, and that the differences between their distributions could be neglected.

(2) We assumed the extents of dendritic fields of individual MNs were sufficiently small relative to the distribution of MN somata within a pool (Cruce, $1974 \mathrm{~b}$; Rosenthal and Cruce, 1985) that the MN soma distribution was the dominant topographic factor.

(3) We neglected the mediolateral dimension of the pools, concentrating on the rostrocaudal distributions of appendicular MNs (Cruce, 1974b).

(4) We assumed the MNs within a pool $\mu$ had a Gaussian distribution of $\rho_{\mu}(x)$ about a position $t_{\mu}$ in the cord, which is given by

$$
\rho_{\mu}(x)=\frac{1}{\sqrt{2 \pi \sigma^{2}}} e^{\left(\left(t_{\mu}-x\right) / 2 \sigma_{\mu}^{2}\right)^{2}}
$$

where $t_{\mu}$ is the center of the MN pool's distribution in the cord and $\sigma_{\mu}$ is the SD of the MN pool distribution in the cord.

(5) We assumed that the density of uniformly distributed cells that would be recruited around the electrode at a position $x$ was a Gaussian distribution $\rho_{\epsilon}(\mathrm{x}, \gamma)$ centered on the electrode location $\gamma$ and given by

$$
\rho_{e}(\mathbf{x}, \gamma)=\frac{1}{\sqrt{2 \pi \sigma_{\gamma}^{2}}} e^{\left((\gamma-x) / 2 \sigma_{\gamma}^{2}\right)^{2}}
$$

where $\gamma$ is the electrode location and $\sigma_{\gamma}$ is a current spread measure for the electrode.

The proportion of the Gaussian population of MNs of the $i$ th pool recruited at a specific electrode location was thus given by

$$
\mathcal{C}_{i}(\gamma)=\int_{-\infty}^{\infty} \rho_{\mu}(x) \rho_{e}(x, \gamma) d x
$$

The integral was evaluated over the length of the model spinal cord. $c_{i}(\gamma)$ represents the fraction of the total MN pool activated. Thus, $c_{i}(\gamma)$ is the coefficient with which to multiply an individual muscle force field. By substituting this $c_{i}(\gamma)$ in Equation 17 of the preceding section, we obtain

$$
F(\gamma)=\sum_{i=1}^{M}\left[\left[\int_{-\infty}^{\infty} \rho_{\mu}(x) \rho_{e}(x, \gamma) d x\right] F_{i}(r)\right]+F_{R} .
$$

The parameters $\sigma_{i}$ and $\sigma_{\gamma}$ were chosen to match estimates of motoneuron topography (Cruce, 1974b) and to model a variety of excitation spreads. $\sigma_{\gamma}$ was varied to allow for electrical coupling and other synaptic interactions among MNs.

\section{References}

Alstermark B, Sasaki S (1986) Integration in descending motor pathways controlling the forelimb in the cat. 15 . Comparison of the projection from excitatory $\mathrm{C} 3-\mathrm{C} 4$ propriospinal neurones to different species of forelimb motoneurones. Exp Brain Res 63:543-566.

Asada H, Slotine J-JE (1986) Robot analysis and control. New York: Wiley.

Bekoff A (1985) Development of locomotion in vertebrates: a comparative perspective. In: Comparative development of adaptive skills: evolutionary implications (Gallin ES, ed), pp 57-94. Hillsdale, NJ: Erlbaum.

Bennett MVL, Spray DC (1987) Intracellular communication mediated by gap junctions can be controlled in many ways. In: Synaptic function (Edelman GM, Gall WE, Cowan WM, eds), pp 109-136. New York: Wiley.

Berkinblit MB, Feldman AG, Fukson OI (1989) Wiping reflex in the frog: movement patterns, receptive fields, and blends. In: Visuomotor coordination: amphibians, comparisons, models, and robots (Ewert J-P, Arbib MA, eds), pp 615-630. New York: Plenum.

Bernstein NA (1967) The coordination and regulation of movements. New York: Pergamon.

Bizzi E, Mussa-Ivaldi FA, Giszter SF (1991) Computations underlying the execution of movement: a novel biological perspective. Science 253:287-291.

Bizzi E, Hogan N, Mussa-Ivaldi FA, Giszter SF (1992) Does the nervous system use equilibrium-point control to guide single and multiple joint movements? Bchav Brain Sci, in press.

Buchanan TS, Rovin GP, Rymer WZ (1989) Strategies for muscle activation during isometric torque generation at the human elbow. $J$ Neurophysiol 62:1201-1213

Chepelyugina MF (1947) On the role of afference in active postural movements. PhD thesis, University of Moscow.

Cohen AH (1987) The structure and function of the intersegmental coordinating system of the lamprey central pattern generation for locomotion. J Comp Physiol 160:181-193.

Cohen AH (1988) Evolution of the vertebrate central pattern generator for locomotion. In: Neural control of rhythmic movements in vertebrates (Cohen AV, Rossignol S, Grillner S, eds), pp 129-166. New York: Wiley.

Cruce WLR (1974a) A supraspinal monosynaptic input to hindlimb motor neurons in lumbar spinal cord of the frog, Rana catesbiana. J Neurophysiol 37:691-704.

Cruce WLR (1974b) The anatomical organization of hindlimb motoneurons in the lumbar spinal cord of the frog, Rana catesbiana. J Comp Neurol 153:59-76.

Deleze J, Herve JC (1983) Effect of several uncouplers of cell-to-cell communication on gap junction morphology in mammalian heart. $\mathrm{J}$ Membr Biol 74:203-215.

Ecker A (1971) The anatomy of the frog. NV: Asher and Co., Amsterdam: Vaals. 
Farris JS (1969) On the cophenetic correlation coefficient. Syst Zool 18:279-285.

Farris JS (1973) On comparing the shapes of taxonomic trees. Syst Zool 22:50-54.

Feldman AG (1979) Central and reflex mechanisms of motor control. Moscow: Nauka.

Feldman $\Lambda G$ (1986) Once more on the equilibrium-point hypothesis ( $\lambda$ model) for motor control. J Mot Behav 18:17-54.

Flash T, Mussa-Ivaldi F (1990) Human arm stiffness characteristics during the maintenance of posture. Exp Brain Res 82:315-326.

Fukson OI, Berkinblit MB, Feldman AG (1980) The spinal frog takes into account the scheme of its body during the wiping reflex. Science 209:1261-1263.

Gelfand IM, Orlovsky GN, Shik ML (1988) Locomotion and scratching in tetrapods. In: Neural control of rhythmic movements in vertebrates (Cohen AV, Rossignol S, Grillner S, eds), pp 167-199. New York: Wiley.

Georgopoulos AP, Kettner RE, Schwartz AB (1988a) Primate motor cortex and free arm movements to visual targets in three-dimensional space. I. Relations between single cell discharge and direction of movement. J Neurosci 8:2913-2927.

Georgopoulos AP, Kettner RE, Schwartz AB (1988b) Primate motor cortex and free arm movements to visual targets in three-dimensional space. II. Coding of the direction of movement by a neuronal population. J Neurosci 8:2928-2937.

Giszter SF, McIntyre J, Bizzi E (1989) Kinematic strategies and sensorimotor transformations in the wiping movements of frogs. J Neurophysiol 62:750-767.

Giszter SF, Bizzi E, Mussa-Ivaldi FA (1991a) Motor organization in the frog spinal cord. In: Analysis and modelling of neural systems (Eeckman FH, ed), pp 377-392. Boston, MA: Kluwer.

Giszter SF, Mussa-Ivaldi FA, Bizzi E (1991b) Equilibrium point mechanisms in the spinal frog. In: Visual structures and integrated functions (Arbib MA, Ewert JP, eds), pp 223-237. New York: Springer.

Giszter SF, Mussa-Ivaldi FA, Bizzi E (1992a) Movement primitives in the frog spinal cord. In: Analysis and modelling of neural systems, Vol II (Eeckman FH, ed), in press. Boston, MA: Kluwer.

Giszter SF, Mussa-Ivaldi FA, Bizzi E (1992b) The organization of motor space in the frog spinal cord. Exp Brain Res Series 22. Control of arm movement in space: neurophysiological and computational approaches (Caminiti R, Johnson PB, Burnod Y, eds). Berlin: Springer-Verlag.

Glusman S, Vazquez G, Rudomin P (1976) Ultrastructural observations in the frog spinal cord in relation to the generation of primary afferent depolarization. Neurosci Lett 2:137-145.

Goldstein H (1981) Classical mechanics. Reading, MA: Addison-Wesley.

Gordon AD (1981) Classification methods for the exploratory analysis of multivariate data. New York: Chapman and Hall.

Gray J, Lissman HW (1940) The effect of deafferentation upon the locomotory activity of amphibian limbs. J Exp Biol 17:227-236.

Gray J, Lissman HW (1946) Further observations on the effect of deafferentation upon the locomotory activity of amphibian limbs. J Exp Biol 23:121-132.

Grillner S, Wallen P (1985) Central pattern generators for locomotion, with special reference to vertebrates. Annu Rev Neurosci 8:233-261.

Grillner S, Zangger P (1979) On the central generation of locomotion in the low spinal cat. Exp Brain Res 34:241-261.

Grobstein P (1990) Strategies for analyzing complex organization in the nervous system. II. A case study: directed movement and spatial representation in the frog. In: Computational neuroscience (Schwartz EL, ed), pp 242-255. Cambridge, MA: MIT Press.

Gustafsson B, Jankowska E (1976) Direct and indirect activation of nerve cells by electrical pulses applied extracellularly. J Physiol (Lond) 258:33-61.

Hering HE (1896) Ueber Devegungsstörungen macht Centripetaler Lähmung. Arch Exp Pathol Pharmakol 38:266-283.

Hogan N (1984) An organizing principle for a class of voluntary movements. J Neurosci 4:2745-2754.

Hogan N (1985) The mechanics of multi-joint posture and movement control. Biol Cybern 52:315-331.

Houk JC (1972) The phylogeny of muscular control configurations. Biocybernetics 4:125-155.

Jhaveri S, Frank E (1983) Central projections of the brachial nerve in bullfrogs: muscle and cutaneous afferents project to different regions of the spinal cord. J Comp Neurol 221:304-312.
Joseph BS, Whitlock DG (1968) Central projections of selected spinal dorsal roots in anuran amphibians. Anat Rec 160:279-288.

Keifer J, Houk JC (1992) NPB technical report 4, Activity-dependent uptake of sulforhodamine labels neural circuits engaged in motor pattern generation in vitro. Chicago: Northwestern University Medical School, Department of Physiology.

Koshland GF, Smith JL (1989) Mutable and immutable features of paw-shake responses after hindlimb deafferentation in the cat. J Neurophysiol 62:162-173.

Lichtman JW, Wilkinson RS, Rich MM (1985) Multiple innervation of tonic endplates revealed by activity-dependent uptake of fluorescent probes. Nature 314:357-359.

Loeb EP, Giszter SF, Borghesani P, Bizzi E (1992) Effects of dorsal root cut on the forces evoked by spinal microstimulation in the frog. $\mathrm{J}$ Somatosensory and Motor Res, in press.

Masino T, Grobstein P (1989) The organization of descending tectofugal pathways underlying orienting in the frog, Rana pipiens. I. Lateralization, parcellation, and an intermediate spatial representation. Exp Brain Res 75:227-244.

Masino T, Knudsen EI (1990) Horizontal and vertical components of head movement are controlled by distinct neural circuits in the barn owl. Nature 345:434-437.

Morgan DL, Proske U (1984) Verbebrate slow muscle: its structure, pattern of innervation, and mechanical properties. Physiol Rev 64: 103-169.

Mortin LI, Keifer J, Stein PSG (1985) Three forms of the scratch reflex in the spinal turtle: movement analyses. J Neurophysiol 53:15011516.

Mussa-Ivaldi FA, Giszter SF (1991) A field-approximation approach to the execution of motor plans. In: Fifth International Conference on Advanced Robotics (ICAR), pp 1200-1204.

Mussa-Ivaldi FA, Bizzi E, Giszter SF (1991a) Transforming plans into actions by tuning passive behavior: a field-approximation approach. Proceedings of the IEEE International Symposium on Intelligent Control (ISIC '91), pp 101-109. New York: IEEE.

Mussa-Ivaldi FA, Giszter SF, Bizzi E (1991b) Motor-space coding in the central nervous system. In: Cold Spring Harbor Symp Quant Biol 55:827-835.

Ostry DJ, Feldman AG, Flanagan JR (1991) Kinematics and control of frog hindlimb movements. J Neurophysiol 65:547-562.

Preparata FP, Shamos MI (1985) Computational geometry. New York: Addison-Wesley.

Ranck JB (1981) Extracellular stimulation. In: Electrical stimulation research techniques (Patterson MM, Kesner RP, eds), pp 2-34. New York: Academic.

Robertson GA, Mortin LI, Keifer J, Stern PSG (1985) Three forms of the scratch refiex in the spinal turtle: central generation of motor patterns. J Neurophysiol 53:1517-1534.

Rosenthal BM, Cruce WLR (1985) The dendritic extent of motoneurons in frog brachial spinal cord: a computer reconstruction of HRPfilled cells. Brain Behav Evol 27:106-114.

Schotland JL, Lee WA, Rymer WZ (1989) Wiping and flexion withdrawal reflexes display different EMG patterns prior to movement onset in the spinalized frog. Exp Brain Res 78:649-653.

Sherrington C (1961) The integrative action of the nervous system. New Haven, CT: Yale UP.

Shik ML, Orlovsky GN (1976) Neurophysiology of locomotor automatism. Physiol Rev 56:465-501.

Simpson JI (1976) Functional synaptology of the spinal cord. In: Frog neurobiology (Llinas R, Precht W, eds), pp 728-749. Berlin: Springer.

Stein PSG (1983) The vertebrate scratch reflex. Symp Soc Exp Biol 37:398-403.

Stein PSG, Mortin LI, Robertson GA (1986) The forms of a task and their blends. In: Neurobiology of vertebrate locomotion (Grillner S, Stein P, Stuart DG, Forssberg H, Herman RM, eds), pp 201-216. London: Macmillian.

von Holst E (1973) The behavioral physiology of animals and man. In: Selected papers of E. von Holst. Coral Gables, FL: University of Miami.

Windhorst UR, Burke RE, Dieringer N, Evinger C, Feldman AG, Hasan Z, Hultborn H, Illert M, Lundberg AP, Macpherson JM, Massion I, Nichols TR, Schwartz M, Vilis T (1991) Group report: what are the units of motor behavior and how are they controlled? In: Motor control: concepts and issues (Humphrey DR, Freund H-J, eds), pp 101-120. New York: Wiley.

Yeomans JS (1990) Principles of brain stimulation. Oxford: Oxford UP. 